\title{
Fate decision of satellite cell differentiation and self-renewal by miR- 31-IL34 axis
}

\author{
Yang Su${ }^{1} \cdot$ Yingying $\mathrm{Yu}^{1} \cdot$ Chuncheng $\mathrm{Liu}^{1,2} \cdot$ Yuying Zhang ${ }^{1} \cdot$ Chang Liu ${ }^{1} \cdot$ Mengxu Ge ${ }^{1} \cdot$ Lei Li $^{1}$ - Miaomiao Lan ${ }^{1}$.

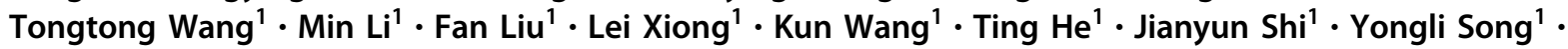 \\ Yaofeng Zhao ${ }^{1} \cdot$ Ning $\mathrm{Li}^{1} \cdot$ Zhengquan $\mathrm{Yu} \mathbb{D}^{1,3} \cdot$ Qingyong Meng $\mathbb{D}^{1,3}$
}

Received: 29 December 2018 / Revised: 13 June 2019 / Accepted: 26 June 2019 / Published online: 22 July 2019

(C) The Author(s) 2019

\begin{abstract}
Quiescent satellite cells (SCs) that are activated to produce numerous myoblasts underpin the complete healing of damaged skeletal muscle. How cell-autonomous regulatory mechanisms modulate the balance among cells committed to differentiation and those committed to self-renewal to maintain the stem cell pool remains poorly explored. Here, we show that miR-31 inactivation compromises muscle regeneration in adult mice by impairing the expansion of myoblasts. miR-31 is pivotal for SC proliferation, and its deletion promotes asymmetric cell fate segregation of proliferating cells, resulting in enhanced myogenic commitment and re-entry into quiescence. Further analysis revealed that miR-31 posttranscriptionally suppresses interleukin 34 (IL34) mRNA, the protein product of which activates JAK-STAT3 signaling required for myogenic progression. IL34 inhibition rescues the regenerative deficiency of miR-31 knockout mice. Our results provide evidence that targeting miR-31 or IL34 activities in SCs could be used to counteract the functional exhaustion of SCs in pathological conditions.
\end{abstract}

These authors contributed equally: Yang Su, Yingying Yu

These authors jointly supervised this work: Zhengquan Yu, Qingyong Meng

\section{Edited by M. Piacentini}

Supplementary information The online version of this article (https:// doi.org/10.1038/s41418-019-0390-x) contains supplementary material, which is available to authorized users.

Qingyong Meng

qymeng@cau.edu.cn

1 State Key Laboratories for Agrobiotechnology, College of Biological Sciences, China Agricultural University, Yuanmingyuan West Road No. 2, Haidian District, Beijing 100193, China

2 The Institute of Bioengineering and Technology, Inner Mongolia University of Science and Technology, Baotou 014010, China

3 Beijing Advanced Innovation Center for Food Nutrition and Human Health, College of Biological Sciences, China Agricultural University, Yuanmingyuan West Road No. 2, Haidian District, Beijing 100193, China

\section{Introduction}

The regeneration, maintenance, and growth of skeletal muscle mainly rely on a small population of muscle stem cells, termed satellite cells (SCs), which reside between the sarcolemma and the basal lamina in a quiescent state [1-3]. When SCs respond to injury, they are rapidly activated to produce thousands of proliferating SCs and then differentiate into myoblasts, which ultimately fuse with preexisting myofibers or each other to repair damaged muscle. A small population of activated SCs can also reverse to quiescence to replenish the stem cell pool, and this process is known as self-renewal [4-6]. During skeletal muscle regeneration, SCs that differentiate to form new fibers and that self-renew to maintain the stem pool must strike a delicate balance [7-11]. Premature differentiation of SCs leads to a reduction in cell number and failure of muscle regeneration [9, 12, 13]. Abnormal stem cell pool maintenance impairs the homeostasis of skeletal muscle and muscle regeneration and results in skeletal muscle performance dysfunction [14, 15].

JAK-STAT3 signaling is an evolutionarily conserved signaling pathway activated by diverse growth factors, cytokines, interferons, and related molecules, and it regulates a variety of biological events ranging from embryonic development to inflammation to the development of several 
types of cancer in humans [16-18]. In skeletal muscle stem cells, JAK-STAT3 signaling was reported to promote terminal myogenic differentiation to the detriment of their proliferation [19]. Inhibition of STAT3 function in the suspended myofiber model increases the planar division of SCs. JAK-STAT3 signaling in SCs increases with age and leads to a decline in cell function through stimulation of asymmetric cell division [20]. All of these findings indicated that JAK-STAT3 activity in SCs has an important role in modulating cell fate segregation; however, the upstream effectors that control the activity of JAK-STAT3 signaling in adult proliferating SCs during muscle regeneration remain poorly understood.

JAK-STAT3 is also involved in processes such as immunity, cell division, cell death, and tumor formation [21-24]. Some interleukin (IL) family members were identified to be regulators of the JAK-STAT3 signaling pathway [16]. A newly discovered member of the IL family, named IL34, which was identified from a comprehensive survey of human proteins in a human monocyte viability assay [25], has a vital role in the regulation of tissueresident monocytes and macrophages [26]. However, until now, there has been no report on the expression and functional analysis of IL34 in the skeletal muscle system.

MicroRNAs (miRNAs) regulate many cellular processes of SCs, including proliferation, differentiation, re-entry into quiescence, and maintenance of quiescence [27-30]. In resting skeletal muscle, miR-31 sequesters Myf5 into mRNA granules, thereby holding quiescent SCs that are poised to enter the myogenic program [31]. A microarray analysis revealed that the expression level of miR-31 was further upregulated in activated SCs relative to quiescent SCs [32]. Profile analysis of Duchenne muscular dystrophy compared with wild-type (WT) muscles revealed that miR31 showed a 50-fold enrichment relative to control samples [33]. All of these findings indicated that miR-31 plays important roles in regulating activated SCs.

In this study, we show that mice with miR-31 deletion have deficient skeletal muscle repair in response to $1.2 \%$ $\mathrm{BaCl}_{2}$ injury. Inefficient regeneration results from the impaired expansion of SCs and relates to the dysregulation of IL34, a cell-autonomous positive regulator of JAK-STAT3 signaling, which impairs the function of SCs through the stimulation of asymmetric division.

\section{Results}

\section{Inefficient skeletal muscle regeneration in miR-31- knockout (KO) mice}

We induced tibialis anterior (TA) muscle injury by injection of $1.2 \% \mathrm{BaCl}_{2}$ and examined the expression profile of
miR-31 during skeletal muscle regeneration. Results showed that miR-31 was dramatically upregulated in regenerating muscles and peaked at day 5 after TA muscle injury (Fig. 1a). In situ hybridization analysis showed that miR-31 is highly present in regenerating TA muscle (Fig. 1b).

Because miR-31 is preferentially expressed in regenerating skeletal muscle, we sought to explore the role of miR31 during skeletal muscle regeneration. We found that inactivation of miR-31 in mice did not affect the whole body weight or wet weight of normal TA muscle (Fig. 1c, d). The myofiber cross-sectional size distribution of uninjured TA muscle also showed no change between WT and miR-31-KO mice (Supplementary Fig. S1a, b). Interestingly, the regenerative capability of TA muscle was markedly reduced in miR-31-KO mice compared with that in WT littermates (Fig. 1e), as shown by an apparent reduction in the size of myofibers with centralized nuclei in miR-31KO mice compared with WT littermates after 5 days of injury (Fig. 1f). Moreover, the percentage of regenerating fibers including two or more centralized nuclei was also significantly reduced in miR-31-KO mice compared with that in WT mice (Fig. 1g). A deficiency in muscle regeneration in miR-31-KO mice was also clearly evidenced by the myofiber size calculation at day 7 after injury (Supplementary Fig. S1a, c). The embryonic/developmental isoform of $\mathrm{MyHC}^{+}(\mathrm{eMyHC})$ fiber size of day 5 post injury TA muscle was remarkably reduced in miR-31-KO mice compared with WT mice (Fig. 1h, i).

After recovery of the TA muscle for 60 days, there was no difference of injured TA muscle between miR-31-KO and WT mice (Supplementary Fig. S1d, e). After a second round of injury using $\mathrm{BaCl}_{2}$ solution, the impaired skeletal muscle regeneration capability in miR-31-KO mice was even more pronounced (Fig. 1j, k). To further evaluate whether intrinsic loss of miR-31 from SCs dampens their functional capacity to participate in muscle regeneration, we transplanted 250,000 SCs from WT and miR-31-KO mice into 2-day injured TA muscle of adult WT mice (Fig. 11). TA muscles from recipient WT mice transplanted with SCs with miR-31 deletion displayed a clear reduction in muscle regeneration (Fig. 1m, n). These results suggest that miR-31 is essential for the early stage of skeletal muscle regeneration.

\section{Deletion of miR-31 reduces the regenerative SC pool and the proliferative capability of SCs}

The results revealed that the frequency of $\mathrm{Pax} 7^{+} \mathrm{SCs}$ was dramatically reduced in 5-day post injury TA muscle of miR-31-KO mice relative to WT mice (Fig. 2a, b), although the number of $\mathrm{Pax}^{+}$cells on transverse sections of uninjured TA muscle and freshly isolated single myofibers was 
a

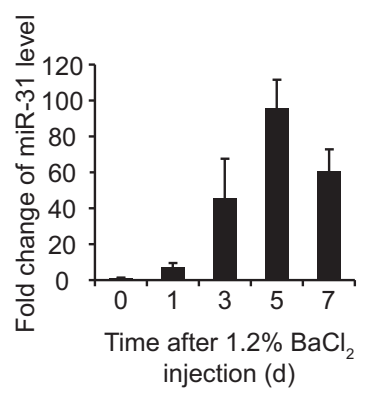

e

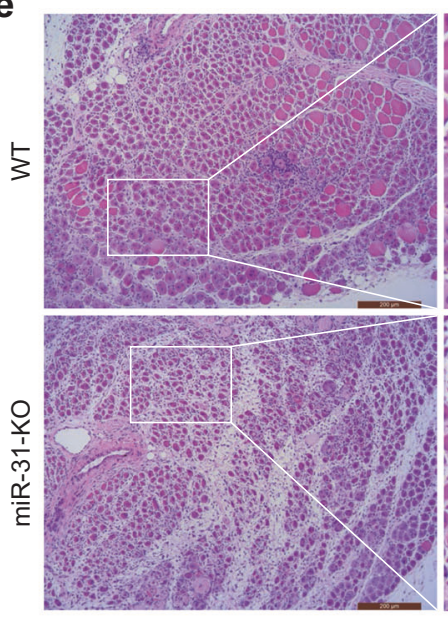

b

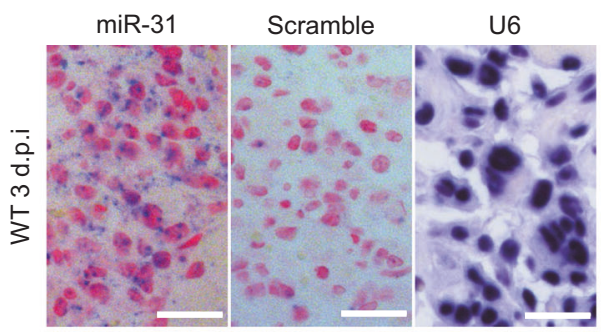

c

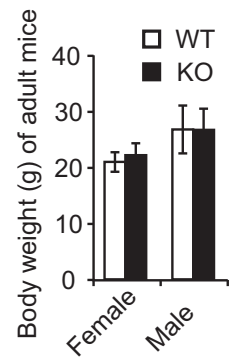

d

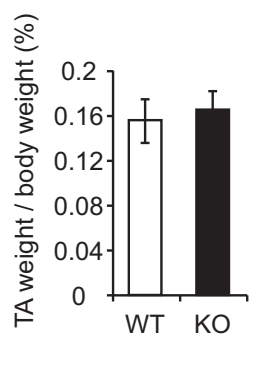

f

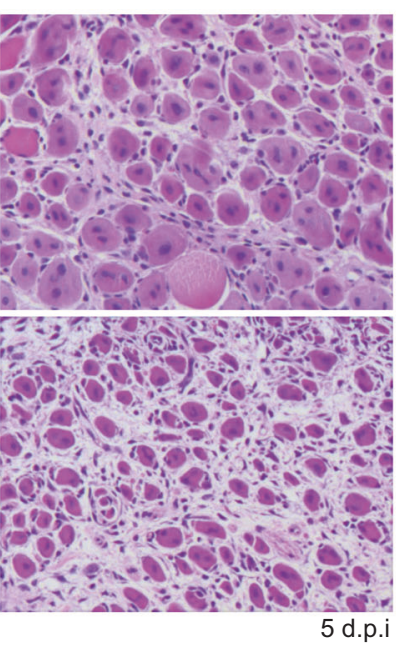

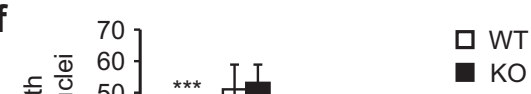

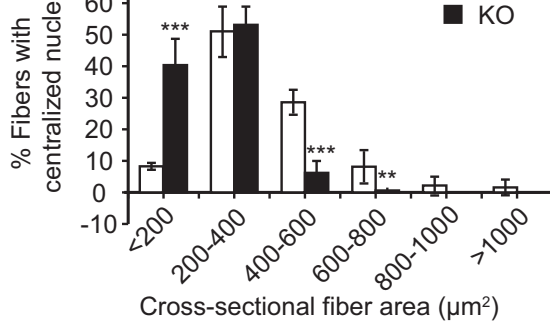

g

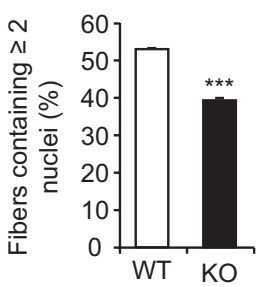

h
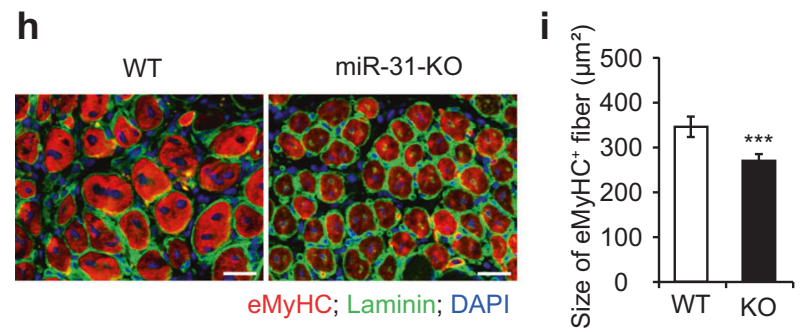

j
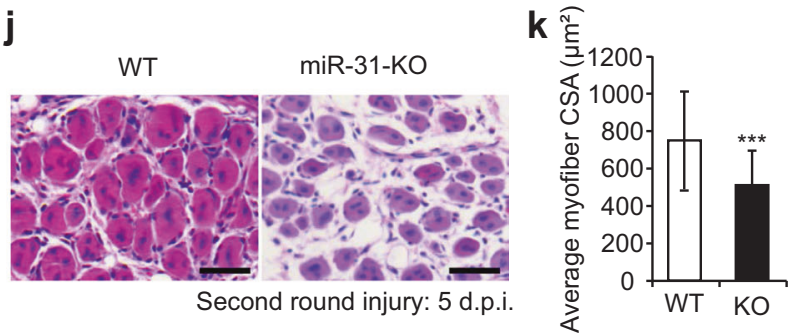

I

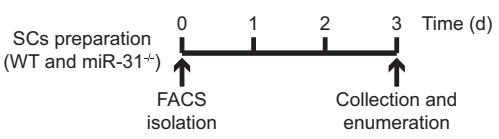

m

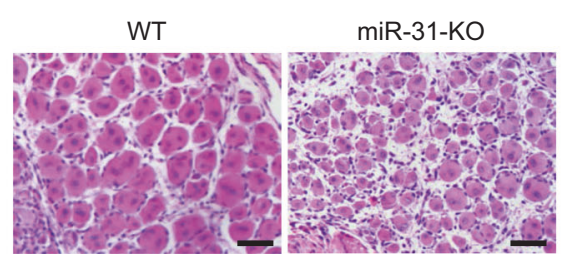

n

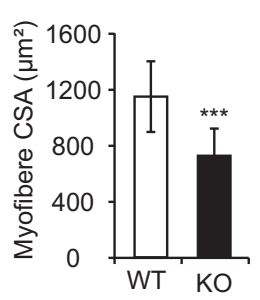

not significantly different between miR-31-KO and WT mice (Supplementary Fig. S2). Remarkably, there were significantly fewer myogenic cells on cultured fibers in miR-31-KO mice than in WT mice (Fig. 2c, d).

We next cultured SCs isolated from the hind limbs of adult WT and miR-31-KO mice in growth medium for
5 days and pulsed them with the thymidine analog EdU for the last $2 \mathrm{~h}$. The proportion of $\mathrm{Pax} 7^{+} \mathrm{EdU}^{+}$cells was significantly lower in miR-31-KO SCs than in WT mice (Fig. 2e, f). The growth rate of plated SCs revealed that SCs without miR-31 had significantly reduced growth potential (Fig. 2g and Supplementary Fig. S3a). To measure whether 
Fig. 1 Deletion of miR-31 in mice impairs skeletal muscle regeneration. a Real-time RT-PCR of miR-31 expression levels in TA muscle from day 0 to day 7 after $\mathrm{BaCl}_{2}$ injury. $\mathbf{b}$ In situ hybridization of miR31 in regenerating skeletal muscle 3 days post injury. Scale bar: $20 \mu \mathrm{m}$. c Absolute body weight of adult WT and miR-31-KO mice. $\mathbf{d}$ Relative uninjured TA muscle wet weight of adult WT and miR-31-KO mice. e Representative photomicrographs of H\&E-stained sections showing a delayed regeneration of injured TA muscle in miR-31-KO mice compared with that in WT littermates at day $5(N=4)$ after $\mathrm{BaCl}_{2}$ injection. Scale bar: $200 \mu \mathrm{m}$. f Regenerating myofiber size (percentage) distributions of WT and miR-31-KO TA muscle 5 days after $\mathrm{BaCl}_{2}$ mediated injury were measured by using ImageJ software. Only myofibers that contained centrally located nuclei were counted. $* * P<$ $0.01 ; * * * P<0.001$. g Quantification of the ratio of regenerating myofibers containing two or more centralized nuclei per field at day 5 post injury. $N=4$ in each group. $* * * P<0.001$. h Representative overlaid photomicrographs of TA muscle sections of WT and miR-31$\mathrm{KO}$ mice 5 days post injury after immunostaining for eMyHC (red) and laminin (green). Nuclei were labeled by DAPI. Scale bar: $30 \mu \mathrm{m}$. i Average CSA of eMyHC-positive fibers in TA muscle 5 days post injury. $* * * P<0.001$. j Sixty days after the first injury, a second injection of $\mathrm{BaCl}_{2}$ solution was delivered to the muscle of WT and miR-31-KO mice, and the muscle was analyzed at day 5 after the second injury. An H\&E-stained TA muscle section from a miR-31-KO mouse presenting severely delayed muscle repair after being subjected to a second injury. Scale bar: $30 \mu \mathrm{m}$. k Quantitative analysis of the average CSA of regenerating myofibers at day 5 after the second injury. $N=3$ in each group. ${ }^{*} * P<0.001$. I Experimental procedure presenting SC preparation and transplantation into the regenerating TA muscle of WT mice. $\mathbf{m}$ Histology analysis of the 5-day recovered TA muscles of WT mice transplanted with SCs by performing H\&E staining. Scale bar: $50 \mu \mathrm{m}$. n Quantification of the average myofiber CSA of regenerating TA muscles transplanted with SCs of adult WT mice 5 days after injury. $N=3$ in each group. $* * * P<0.001$

the mitogenic ability of SCs was attenuated by loss of miR31 function, we calculated the elapsed time of dividing cells undergoing two rounds of the cell cycle by tracking FASCisolated cells using videomicroscopy (Supplementary Movie. S1, 2). We observed no clear difference in the elapsed time of dividing cells undergoing twice the number of cell divisions from the 2nd to the 3rd cell cycle (Supplementary Fig. S3b).

To investigate the proliferating potential of SCs during muscle regeneration, we used immunohistochemistry to detect $\mathrm{Ki}^{+} 7^{+}$cells in regenerating skeletal muscle, in which numerous proliferating and differentiating cells are normally present [34], at day 3. Indeed, the number and frequency of $\mathrm{Ki} 67^{+}$cells on transverse sections of TA muscle were dramatically reduced in miR-31-KO mice relative to WT mice (Fig. $2 \mathrm{~h}-\mathrm{j}$ ). EdU was also added to stain proliferating cells for the last $2 \mathrm{~h}$ before sacrificing the mice, and the number of $\mathrm{EdU}^{+}$cells was found to be significantly reduced in miR-31KO mice compared with that in WT mice (Supplementary Fig. S3c, d). To identify SC-associated proliferating myoblasts, anti-MyoD, and anti-Ki67 antibodies were used to coimmunostain transverse sections of day 3 post injury TA muscle [35]. miR-31 inactivation resulted in a significant decrease in $\mathrm{MyoD}^{+} \mathrm{Ki}^{+} 7^{+}$cells (Fig. 2k, 1). Interestingly, the protein level of MyoD was drastically increased in the day 3 post injury TA muscle of miR-31-KO mice compared with that in WT mice (Fig. 2m), highlighting the possibility that miR-31-deficient proliferating SCs are more inclined to differentiate.

\section{Inactivation of miR-31 promotes the myogenic lineage progression of SCs}

To detect the myogenic fate decisions of miR-31-deficient SCs, fluorescence-activated cell sorting (FACS)-isolated SCs from the hind limbs of adult WT and miR-31-KO mice were cultured and then induced to differentiate to form new myotubes. Committed SCs $\left(\mathrm{MyoD}^{+} \mathrm{Ki}^{-} 7^{-}\right)$occupied a significantly higher proportion among SCs lacking miR-31 relative to corresponding WT SCs plated in growth medium (Supplementary Fig. S4a, b). Interestingly, a dramatically higher proportion of $\mathrm{MyoD}^{+} \mathrm{Ki}^{-} 7^{-}$cells was also found in miR-31-KO SCs at day 1 after differentiation (Fig. 3a, b). Consistent with the immunostaining results, MyoD and MyoG protein levels were significantly increased in miR31-KO cultures (Fig. 3c). After 2 days in differentiation medium, miR-31 inactivation promoted terminal myogenic differentiation of SCs, as shown by a higher differentiation index relative to WT SCs (Fig. 3d, e). The frequency of undifferentiated proliferating $\mathrm{Ki}^{+} 7^{+}$cells was significantly reduced in miR-31-KO cell cultures (Fig. 3f). Moreover, miR-31 inactivation increased the ratio of both the selfrenewing $\mathrm{Pax}^{+} \mathrm{MyoD}^{-}$and differentiated $\mathrm{Pax}^{-} \mathrm{MyoD}^{+}$ cell populations and decreased the ratio of the proliferating Pax $7^{+}$MyoD $^{+}$cell population (Fig. 3g, h).

\section{Loss of miR-31 drives $\mathrm{Pax7}^{+}$proliferating SCs into quiescence}

Inactivation of miR-31 increased the ratio of the $\mathrm{Pax} 7^{+} \mathrm{MyoD}^{-}$cell population (Fig. 3h), leading us to examine whether miR-31 also controls the re-entry of SCs into quiescence during skeletal muscle regeneration and in vitro culture. Consistently, deletion of miR-31 in mice severely increased the percentage of $\mathrm{Pax} 7^{+} \mathrm{Ki} 67^{-}$quiescent cells in TA muscle after injury at day 5 (Fig. 4a, b), on which numerous $\mathrm{Pax}^{+}$cells replenish the satellite stem cell pool [36]. Consistent with reports demonstrating that quiescent SCs express a higher level of Pax7, we indeed detected a significant increase in both the mRNA and protein levels of Pax7 in day 5 post injury skeletal muscle of miR31-KO mice compared with that of WT mice (Fig. 4c, d).

FACS-isolated SCs from the hind limb muscle of miR$31 \mathrm{KO}$ and WT mice were first cultured in growth medium and then induced to differentiate. We detected that miR-31 deletion resulted in a dramatically higher proportion of noncycling $\mathrm{Pax}^{+} \mathrm{Ki}^{-} 67^{-}$SCs (Fig. 4e-h). The niche has a 
a

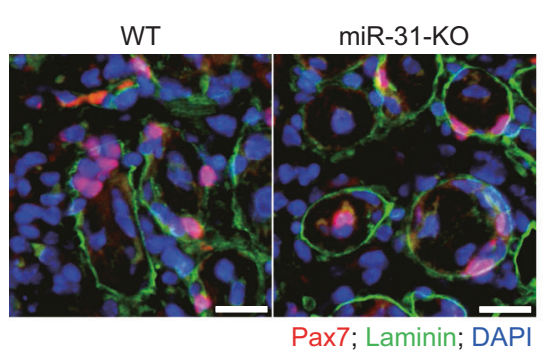

e

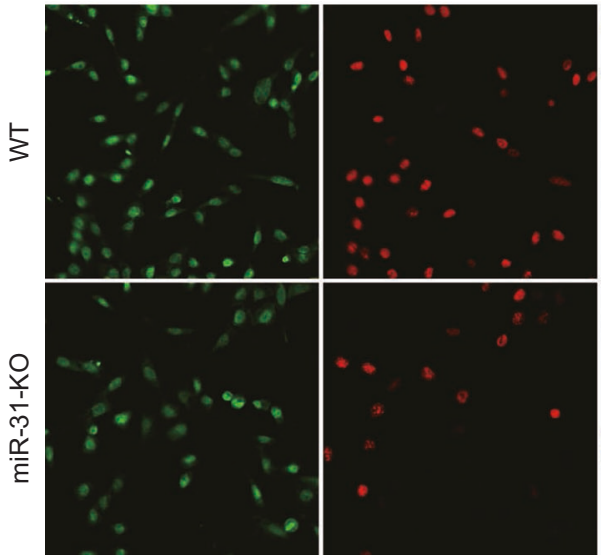

h

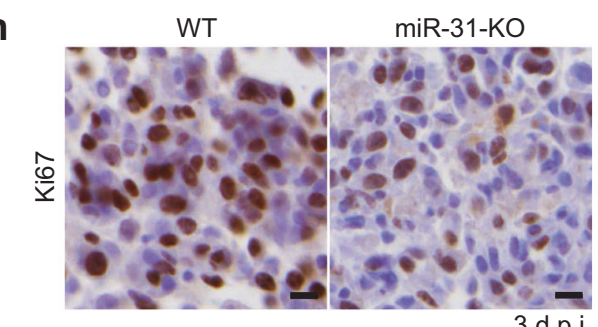

$\mathbf{k}$

k $\quad$ MyoD

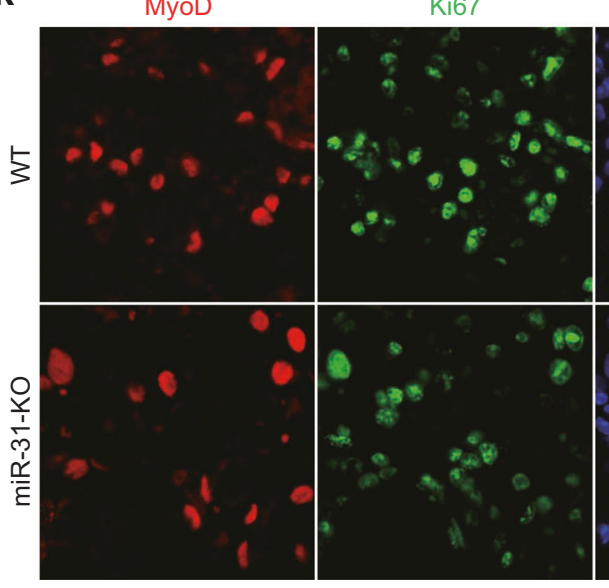

\section{b}

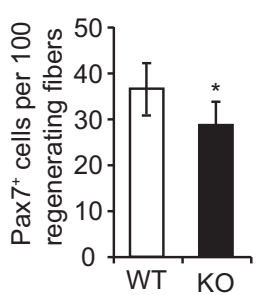

DAPI

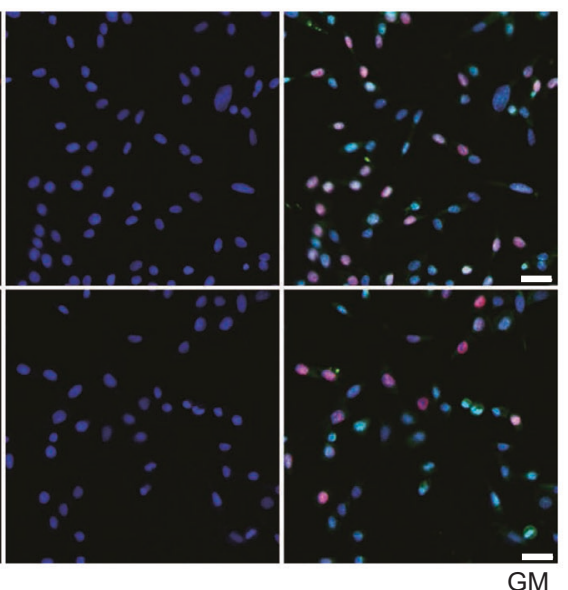

i

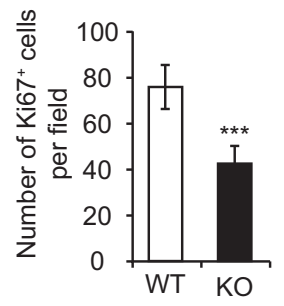

DAPI

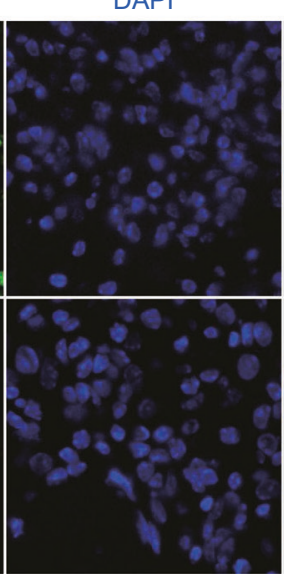

C

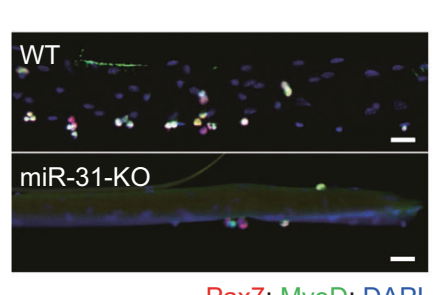

Pax7; MyoD; DAPI d

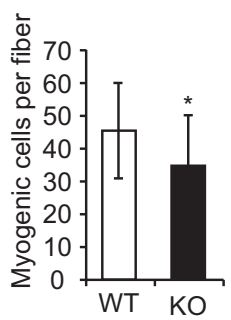

f

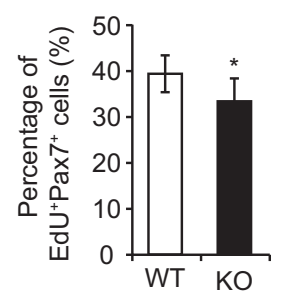

g

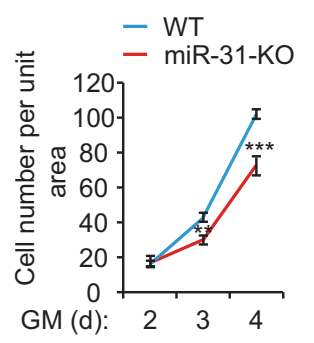

j

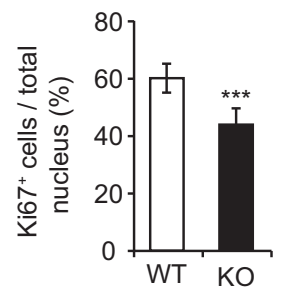

I

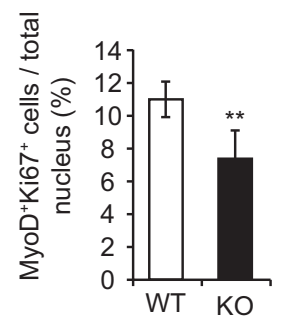

m

WT KO WT KO KDa

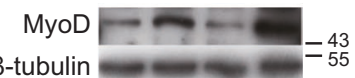

key role in regulating the cell fate of SCs [10]. To examine whether SC re-entry into quiescence is affected by the niche, we examined the cell fate of SCs on cultured single myofibers. Intriguingly, we detected a dramatic increase in the proportion of self-renewing $\mathrm{Pax}^{+} \mathrm{MyoD}^{-}$cells in clusters of mice with miR-31 deletion (Fig. $4 \mathrm{i}, \mathrm{j}$ ). 
Fig. 2 miR-31 is required for the maintenance of a regenerative SC pool and the proliferation of SCs. a Representative merged photomicrographs of day 5 post injury transverse sections of TA muscle stained for Pax7 and laminin from WT and miR-31-KO mice. Nuclei were labeled by costaining with DAPI. Scale bars: $20 \mu \mathrm{m}$. b Quantification of the number of $\mathrm{Pax} 7^{+}$cells per regenerating myofiber of WT and miR-31-KO mice. $* P<0.05$. c Representative merged photomicrographs of $72 \mathrm{~h}$ cultured myofibers from WT and miR-31-KO mice costained with MyoD, Pax7, and DAPI. Scale bars: $30 \mu \mathrm{m}$. d Quantification of myogenic cell number per myofiber. $* P<0.05$. e SCs were established from WT and miR-31-KO mice. Cells were cultured in growth medium, and EdU was added to label proliferating cells for the last $2 \mathrm{~h}$. The cells were then fixed and analyzed by staining with Pax7, EdU, and DAPI. Representative individual and merged photomicrographs of WT and miR-31-KO cultures after labeling with EdU, Pax7, and DAPI. Scale bars: $30 \mu \mathrm{m}$. f Quantitative analysis of the frequency of $\mathrm{Pax}^{+} \mathrm{EdU}^{+}$double-positive cells in WT and miR-31-KO cultures. ${ }^{*} P<0.05$. g Quantification of the growth rate of WT and miR-31-KO SCs from day 2 to day 4 in growth medium. $* * P<0.01$; $* * * P<0.001$. h Immunohistochemistry analysis of $\mathrm{Ki}^{+} 7^{+}$cells in regenerating muscle from WT and miR-31-KO mice at day 3 after injury. Scale bars: $20 \mu \mathrm{m}$. i, j Quantification of the number and percentage of $\mathrm{Ki}^{+} 7^{+}$cells in regenerating TA muscle of WT and miR-31$\mathrm{KO}$ mice. $* * * P<0.001$. $\mathbf{k}$ Representative individual and merged images of day 3 post injury TA muscles of WT and miR-31-KO mice; red, MyoD; green, Ki67; blue, DAPI. Scale bar; $20 \mu \mathrm{m}$. I Quantification of the percentage of $\mathrm{MyoD}^{+} \mathrm{Ki}_{67^{+}}$double-positive cells among the total nuclei in injured TA muscle of WT and miR-31-KO mice. $* * P<0.01$. $\mathbf{m}$ Immunoblots presented here demonstrate the MyoD and unrelated $\beta$-tubulin protein levels in day 3 post injury TA muscle of WT and miR-31-KO mice

\section{miR-31 directly targets interleukin 34 (IL34) in proliferating SCs}

To further elucidate the role of miR-31 during myogenesis, we performed a gene expression microarray analysis using WT and miR-31-KO proliferating SCs after incubation in growth medium. From the significant differential gene heatmap, four genes were significantly upregulated in miR31-KO mice compared with WT mice (Fig. 5a). The 3 ' untranslated region (3'-UTR) of IL34, which is remarkably expressed in proliferating SCs of miR-31-KO mice (Fig. 5b, c), was predicted as the target of miR-31 by two target prediction algorithms (miRanda; www.microrna.org/ and TargetScan; www.Targetscan.org/). To further confirm whether miR-31 affects IL34 messenger RNA by interacting with putative target sites in the $3^{\prime}$-UTR, a partial sequence of the IL34-3'-UTR including the miR-31-binding site and a mutated 3'-UTR of IL34 was cloned into a luciferase reporter (Fig. 5d). The constructs were co-transfected with negative control and miR-31 mimic expression constructs into HEK293T cells. miR-31 repressed luciferase activity in the presence of WT constructs, and this repression was abolished by co-transfection with miR-31 and a luciferase reporter including a mutant form of the miR-31-binding site on IL34 (Fig. 5e).

\section{miR-31 prevents hyperactivation of JAK-STAT3 signaling by repressing IL34}

Interleukin family members are important upstream signaling cytokines that mediate activation of the JAK-STAT pathway, leading us to investigate the effects of miR-31 inactivation on the JAK-STAT3 signaling pathway. We found that IL34, STAT3 phosphorylation, and total STAT3 protein levels were all strongly increased in both proliferating and differentiating cultures with loss of miR-31 (Fig. 6a, b). Upregulation of the total STAT3 protein level is a type of autoregulation that has been previously documented [37, 38]. To further investigate the effects of hyperactivation of JAK-STAT3 signaling on the fate of SCs, we isolated and cultured single myofibers for $42 \mathrm{~h}$, the time point at which SCs have just undergone the first cell division, and then fixed the SCs for coimmunostaining with anti-Pax7 and anti-Ki67 antibodies. Cell doublets containing one $\mathrm{Pax} 7^{+} \mathrm{Ki} 67^{-}$cell and one $\mathrm{Pax} 7^{+} \mathrm{Ki} 67^{+}$cell occupied a significantly higher ratio among the dividing cells of miR31-KO mice than among those of WT mice (Fig. 6c, d). Interestingly, WT SC cultures were treated with recombinant OSM, an activator of JAK-STAT3 signaling, to induce tyrosine phosphorylation of STAT3 both under growth and differentiation conditions (Supplementary Fig. S5a). In agreement with the SCs with loss of miR-31 function, OSM treatment led to a subpopulation of $\mathrm{Pax} 7^{+}$cells that reversed to quiescence (Supplementary Fig. S5b, c), enhanced the terminal myogenic differentiation of SCs (Supplementary Fig. S5d, e) and a decreased ratio of the $\mathrm{Ki}^{6} 7^{+}$cell population compared with bovine serum albumin (BSA)-treated control cells (Supplementary Fig. S5f).

We next examined whether knockdown of IL34 messenger RNA could reduce p-STAT3 protein levels in miR31-KO cultures. IL34 and p-STAT3 protein levels were apparently downregulated upon transfection of miR-31deficient proliferating SCs with a lentivirus expressing shRNA that specifically interfered with IL34 (Fig. 6e and Supplementary Fig. S5g). We also treated WT cultures ex vivo with an essential physiological activator of the JAK-STAT3 signaling (interleukin 6 (IL6)) to measure the activation of JAK-STAT3. Notably, treatment with IL6 significantly promoted activation of JAK-STAT3 as did loss of miR-31, suggesting that miR-31-regulated IL34 is an effective axis to stimulate JAK-STAT3 signaling (Supplementary Fig. S5h). Moreover, IL34 siRNA treatment decreased the ratio of the $\mathrm{Ki}^{-} 7^{-}$cell population in miR-31-KO cultures (Fig. 6f, g). To further investigate the effects of impaired IL34 function on the regeneration of skeletal muscle of miR-31-KO mice, we treated injured TA muscle with a lentivirus expressing shRNA that specifically interfered with IL34. IL34 shRNA treatment promoted the 
a
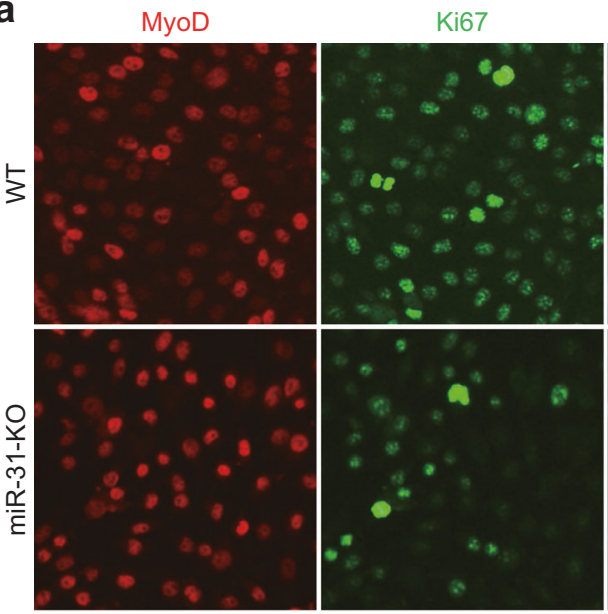

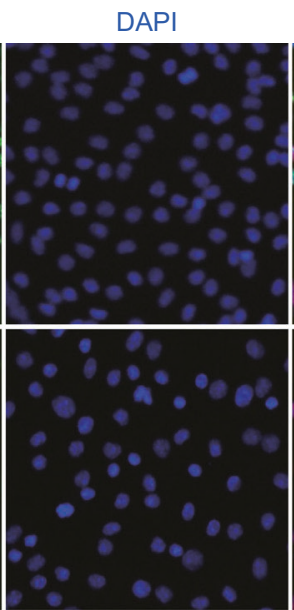

Merged

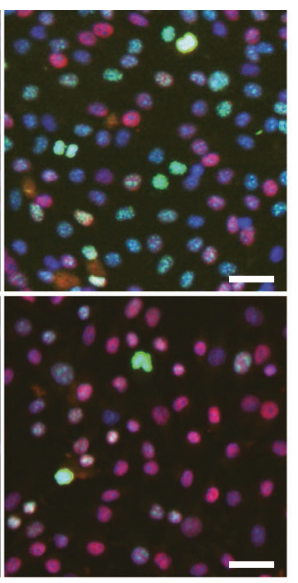

$\mathrm{GM}>\mathrm{DM} 1$ b

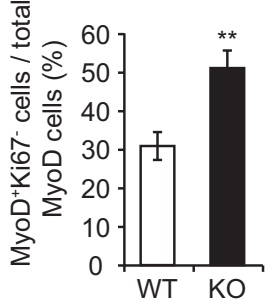

C

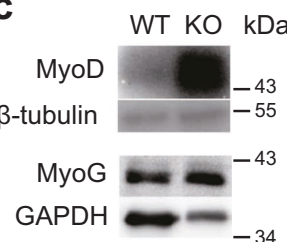

d

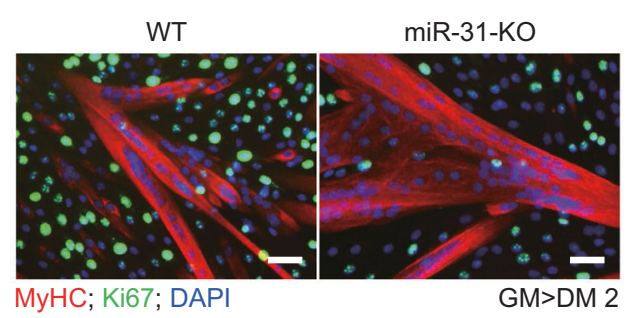

e

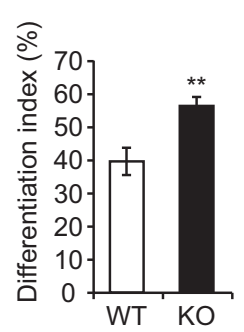

f

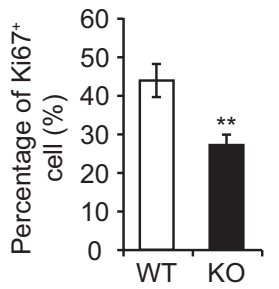

h

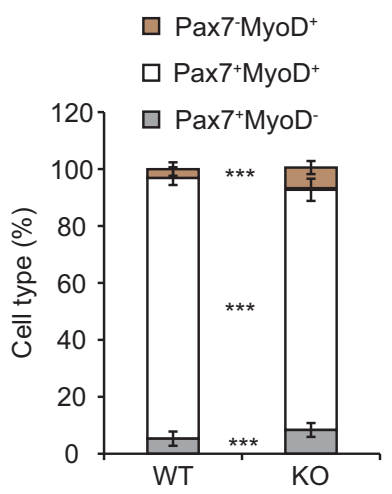

Fig. 3 Inactivation of miR-31 promotes the myogenic lineage progression of SCs. a WT and miR-31-KO SCs were cultured in growth medium for 4 days and then switched to differentiation medium and cultured for 1 day. The cells were then fixed and labeled with antiMyoD and anti-Ki67 antibodies. DAPI was used to identify nuclei. Representative individual and overlaid images of WT and miR-31-KO cultures after labeling with MyoD, Ki67, and DAPI. Scale bars: $30 \mu \mathrm{m}$. b Percentage of $\mathrm{MyoD}^{+} \mathrm{Ki}^{-}$cells (normalized to $\mathrm{MyoD}^{+}$cells) in WT and miR-31-KO cell cultures. ${ }^{*} P<<0.01$. c The representative immunoblots presented here show MyoD and MyoG protein levels in WT and miR-31-KO cultures after undergoing differentiation for 1 day. d After 4 days in growth medium, the cells were induced to differentiate for 2 days. Myogenic differentiation was examined by staining for MyHC. Myogenic proliferation was determined by immunostaining against Ki67. Representative merged images of WT and miR-31-KO cultures after labeling with MyHC, Ki67, and DAPI. Scale bars: $30 \mu \mathrm{m}$. e The differentiation index was determined by the percentage of nuclei in MyHC-positive cells. ${ }^{*} P<0.01$. f Percentage of $\mathrm{Ki}^{+} 7^{+}$cells (normalized to DAPI cells) in WT and miR-31-KO cell cultures. $* * P<0.01$. g SCs isolated from hindlimb muscles of WT and miR-31-KO mice were cultured in growth medium for 5 days. The cells were then fixed and measured by immunostaining against Pax7, MyoD, and DAPI. Representative individual and overlaid images of WT and miR-31-KO SC cultures after labeling with Pax7, MyoD, and DAPI. Scale bar: $30 \mu \mathrm{m}$. h Quantification of the percentages of selfrenewing $\mathrm{Pax}^{+} \mathrm{MyoD}^{-}$, proliferating $\mathrm{Pax}^{+} \mathrm{MyoD}^{+}$and differentiated $\mathrm{Pax}^{-} \mathrm{MyoD}^{+}$cell populations in WT and miR-31-KO cultures. $* * * P$ $<0.001$ 
a

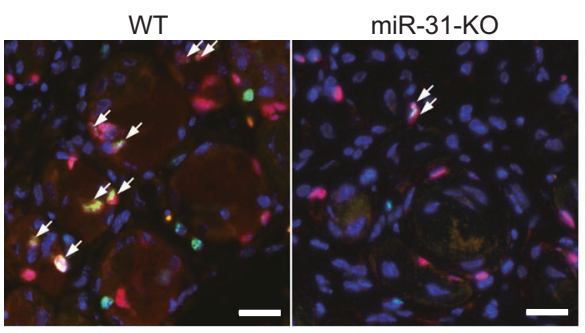

Pax7; Ki67; DAPI b

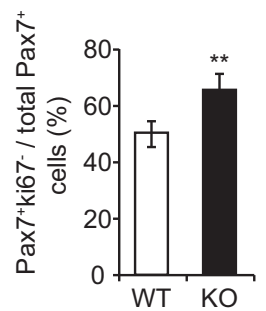

c

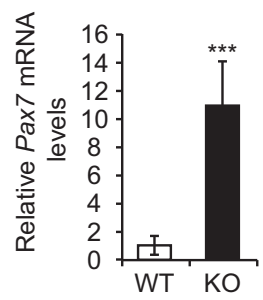

d

WT KO WT KO WT KO kDa

$\mathrm{Pax} 7 \mathrm{~W}=-55$

GAPDH

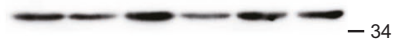

e
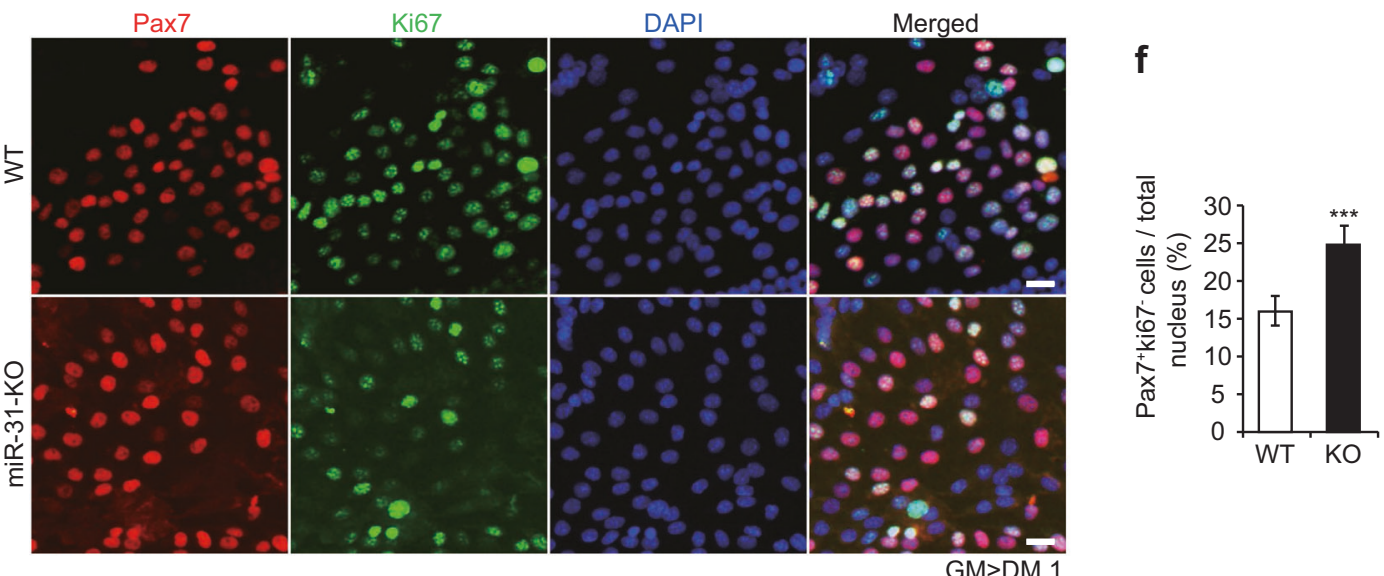

g

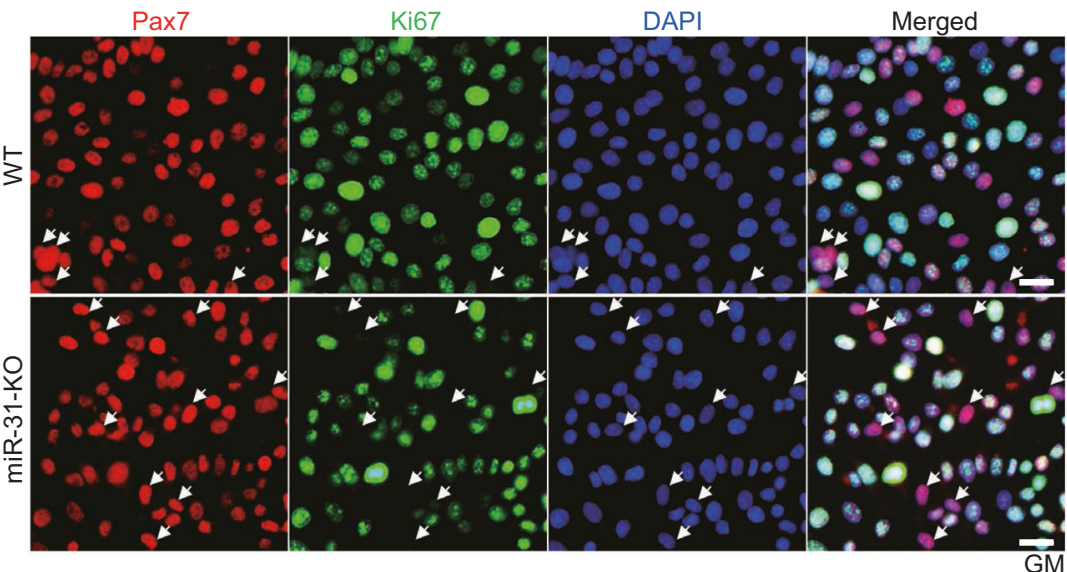

h

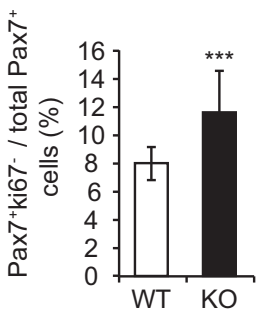

i

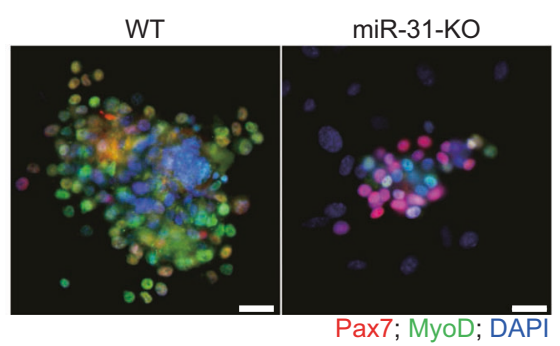

recovery of injured TA muscle of miR-31-KO mice (Fig. 6h, i) and reduced the protein levels of both IL34 and p-STAT3 in the regenerating TA muscle of miR-31-KO mice (Fig. 6j). In addition, we treated regenerating TA j

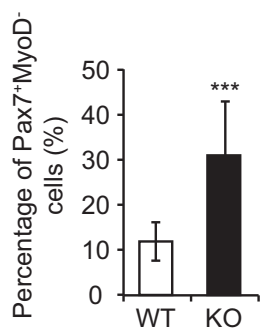

muscles of miR-31-KO mice with the STAT3 inhibitor 5,15 DPP. Remarkably, inhibition of JAK-STAT3 significantly promoted the recovery of injured TA muscles of miR-31KO mice (Fig. 6k, 1). 
Fig. 4 Loss of miR-31 drives Pax7-positive proliferating SCs into quiescence. a Representative photomicrographs of day 5 post injury TA muscle sections of WT and miR-31-KO mice after immunostaining for Pax7 (red) and Ki67 (green). Nuclei were labeled by staining with DAPI. Arrows point to $\mathrm{Pax} 7^{+} \mathrm{Ki} 67^{+}$cells. Scale bar: 20 $\mu \mathrm{m}$. b Quantification of the percentage of $\mathrm{Pax}^{+}$cells that did not coexpress Ki67 among total $\mathrm{Pax} 7^{+}$cells. ${ }^{* *} P<0.01$. c Relative mRNA levels of Pax7 in day 5 post injury TA muscles of WT and miR-31-KO mice measured by qRT-PCR. $* * * P<0.001$. d The immunoblots presented here illustrate the protein levels of $\operatorname{Pax} 7$ and an unrelated GAPDH in TA muscles of WT and miR-31-KO mice at day 5 after $\mathrm{BaCl}_{2}$ injection. e Representative individual and overlaid images of 1day differentiated WT and miR-31-KO SCs after labeling Pax7, Ki67 and DAPI. Scale bars: $30 \mu \mathrm{m}$. f Quantification estimation of the percentage of $\mathrm{Pax} 7^{+} \mathrm{Ki} 67^{-}$quiescent cells in WT and miR-31-KO cultures. $* * * P<0.001$. g Representative individual and overlaid images of WT and miR-31-KO proliferating SCs after labeling Pax7, Ki67 and DAPI. Scale bars: $30 \mu \mathrm{m}$. h Quantification estimation of the percentage of $\mathrm{Pax} 7^{+} \mathrm{Ki} 67^{-}$quiescent cells in $\mathrm{WT}$ and miR-31-KO cultures. $* * * P$ $<0.001$. i Single myofibers were isolated from the extensor digitorum longus (EDL) muscle of WT and miR-31-KO mice. After 5 days of culture, myofiber-derived SC clusters were fixed and stained for Pax7, MyoD, and DAPI. Representative merged photomicrographs of myofiber-derived SC clusters after immunostaining for Pax7 (red) and MyoD (green). Nuclei were identified by labeling with DAPI. Scale bar: $50 \mu \mathrm{m}$. j Percentage of self-renewing $\mathrm{Pax}^{+} \mathrm{MyoD}^{-}$SCs (normalized with DAPI) in WT and miR-31-KO myofiber-derived SC cultures. $* * * P<0.001$

To further explore the impact of IL34 on the JAK-STAT3 pathway, we treated cultured WT SCs with recombinant IL34 to detect the activity of JAK-STAT3 signaling. Importantly, upregulation of $\mathrm{p}$ STAT3 protein levels was detected in cell cultures following IL34 treatment relative to BSA (control) treatment (Fig. 6m). To measure whether forced expression of IL34 in damaged TA muscle of WT mice could impair the repair of skeletal muscle, we utilized the $\mathrm{BaCl}_{2}$-induced muscle regeneration mouse model. We observed remarkably smaller regenerating myofibers in IL34-expressing adenovirus-treated TA muscles at day 5 after injury (Fig. 6n, o). IL34-expressing adenovirus-treated injured TA muscles exhibited higher levels of Pax7, IL34, p-STAT3, and STAT3 than control adenovirus-treated injured TA muscles (Fig. 6p). These results indicate that the miR-31-IL34 axis in proliferating SCs functions in modulating JAK-STAT3 signaling activity to the proper level.

\section{IL34 is required for myogenic lineage progression by activating JAK-STAT3 signaling in cultured SCs}

Although we found increases in IL34 and p-STAT3 protein levels in SCs lacking miR-31, it remains unknown whether IL34 modulates STAT3 activation by a cell-autonomous regulatory mechanism. To address this question, we measured IL34 and p-STAT3 protein levels during in vitro myogenesis using cultured SCs. The increase in the IL34 protein level correlated with an increase in the p-STAT3 level, suggesting that IL34 was a potentially positive regulator of JAK-STAT3 signaling (Fig. 7a). We then infected WT proliferating SCs with a lentivirus expressing shRNA against IL34 and performed a FACS-based assessment to purify stably infected cells, which were cultured and induced to differentiate (Fig. 7b). Treatment with shIL34 caused a significant decrease in IL34 and p-STAT3 protein levels (Fig. 7c) and a reduction in Pax7, MyoD, and MyHC protein levels (Fig. 7d). Infection with shIL34 led to the persistent existence of a high percentage of both $\mathrm{Pax} 7^{+}$and $\mathrm{MyoD}^{+}$cells (Fig. 7e-h). However, these cells were unable to initiate the expression of myogenin (Fig. 7i, j). We also demonstrated that loss of IL34 function impaired terminal myogenic differentiation of SCs, as shown by a lower differentiation index than that produced by transfection with shScramble (Fig. 7k, 1). Collectively, these results indicated that IL34 is an intrinsic regulator that initiates myogenic progression via activation of JAK-STAT3 signaling.

\section{Discussion}

Previous studies have reported that the transcription level of miR-31 in activated SCs is upregulated [32], indicating that miR-31 is pivotal for the cell-autonomous regulatory mechanisms of activated SCs, which is in line with our in situ hybridization and qRT-PCR analyses, both of which further revealed that miR-31 was upregulated in injured muscle (Fig. 1a, b). In this study, we showed that $\mathrm{BaCl}_{2}$ induced muscle regeneration was severely delayed at the early stage in mice with loss of miR-31 (Fig. 1e). This defective regeneration was mainly ascribed to the SCspecific loss of function of miR-31, which was supported by transplanting WT and miR-31-KO SCs into injured TA muscles of WT mice (Fig. 11-n). Although miR-31 deletion had no effect on satellite stem pool at basal line, there was a dramatic reduction in $\mathrm{Pax}^{+}$cell numbers in both regenerating myofibers and in vitro cultured single myofibers. These results suggested that miR-31 is important for the function of SCs in their activated phase. Results showed that the proliferation capacity of SCs in regenerating muscle and cultured SCs was reduced in miR-31-KO mice. Interestingly, we observed that deletion of miR-31 increased the proportion of $\mathrm{Pax} 7^{+} \mathrm{Ki} 67^{-}$quiescent cells as well as the percentage of $\mathrm{Myod}^{+} \mathrm{Ki}^{-} 7^{-}$committed cells. The results suggest that the reduction of cycling SCs is attributed to asymmetric cell fate segregation, which generates one differentiated cell and one quiescent stem cell. Upregulation of miR-31 has been shown to decrease the activity of both endothelial and neuronal nitric oxide synthase (NOS), which participate in muscle repair by modulating SC functions [39-41]. Inhibition of nitric oxide signaling in miR-31-KO cultures by treatment with an NOS inhibitor, L- 


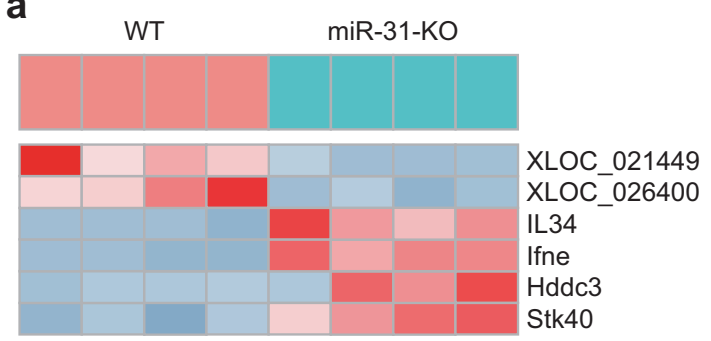

d

Position 383-389 of IL34 3'-UTR

IL34 3'-UTR 5'...CAACCAAAUGCCGUAUCUUGCCA...3

mmu-miR-31 3'...GUCGAUACGGUCGUAGAACGGA...5

Position 383-389 of IL34 3'-UTR

IL34 mut-UTR 5'...CAACCAAAUGCCGUAAGCACUAA...3' | | | | |

mmu-miR-31

3'...GUCGAUACGGUCGUAGAACGGA...5

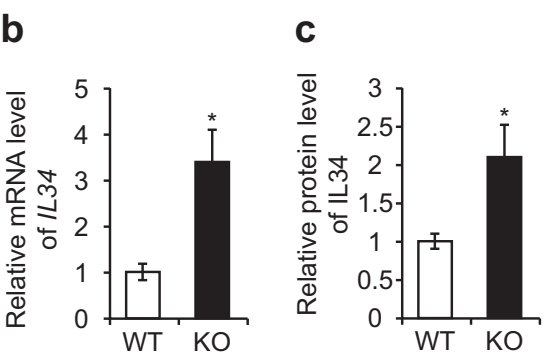

e

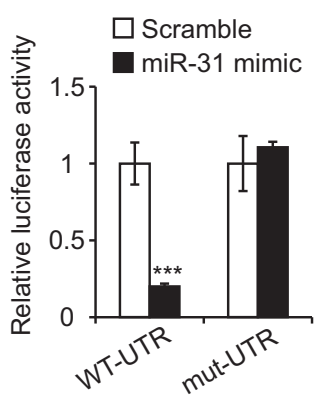

Fig. 5 miR-31 targets IL34 in proliferating SCs. a Heatmaps of selected up- and downregulated genes of proliferating SCs after inactivation of miR-31. b Relative mRNA levels of IL34 in cultured proliferating SCs isolated from WT and miR-31-KO mice measured with a qRT-PCR assay. $* P<0.05$. c Densitometry analysis of immunoblots for IL34 levels in WT and miR-31-KO proliferating SCs. $N=$ 4 for the western blot and densitometry analyses. $* P<0.05$. d

NAME, resulted in weak changes in cell functions (Supplementary Fig. S6), indicating that miR-31-regulated skeletal muscle regeneration has little relation to nitric oxide signaling. Collectively, the cell-autonomous regulatory mechanism of miR-31 to balance SC differentiation and self-renewal underscores its vital role in effectively sustaining skeletal muscle regeneration.

Analysis of the molecular mechanisms revealed that miR-31 promotes the expansion of proliferating SCs by repressing IL34, a cytokine produced by a wide range of cells. IL34 is highly conserved among all vertebrates from fish to humans, and it is associated with a series of inflammatory diseases and tumorigenesis [42-45]. These results suggest that IL34 modulates many biological developmental and pathological processes. However, until now, the expression and functional analysis of IL34 in the regulation of the skeletal muscle system has not been elucidated. Our RNA-seq screening detected the dramatic upregulation of IL34 in activated SCs lacking miR-31, which directly binds to the 3 '-UTR of IL34 mRNA, indicating miR-31-mediated IL34 is essential for SC functions.

Asymmetric division, one of the important characteristics of muscle stem cells, is regulated by a cooperation of several complex signaling $[46,47]$. Reports have demonstrated that elevated JAK-STAT3 signaling in aging or diseased SCs leads to cells preferentially undergoing asymmetric cell fate segregation, resulting in impaired muscle regeneration
TargetScan predicted the potential miR-31 target site to be in the $3^{\prime}$ UTR of IL34. The nucleotide coordinate of IL34 was in accordance with that in the mouse Refseq database (NM_001135100.2). Mutations from the seed sequence are indicated. e Relative luciferase activity in $293 \mathrm{~T}$ cells transfected with constructs of luciferase cDNA ligated to the 3'-UTR or the mutated 3'-UTR of IL34 messenger RNAs with miR-31 mimics and negative control. $* * * P<0.001$

and gradually exhausting the satellite stem pool $[8,20,48,49]$. Our results provide the first genetic evidence that IL34 is a positive intrinsic cellular modulator of JAK-STAT3 signaling and is required for myogenic lineage progression. Loss of function of miR-31-mediated upregulation of IL34 facilitating JAK-STAT3 activity compromised SC function during skeletal muscle regeneration. Stable knockdown of IL34 in WT SCs resulted in the failure of terminal differentiation of $\mathrm{Pax}^{+} \mathrm{MyoD}^{+}$cells by repressing JAK-STAT3 signaling activity. Forced treatment of WT adult SCs with IL34, similar to loss of miR-31, resulted in overactivated JAK-STAT3 signaling. Hyperactivation of JAK-STAT3 signaling by treatment of WT adult proliferating SCs with Oncostatin M (OSM) resulted in a population of $\mathrm{Pax}^{+}$cells that reversed into quiescence (Supplementary Fig. S5b, c), supporting previous descriptions that OSM secreted by muscle fibers is responsible for the quiescence induction of muscle stem cells [50], and the other cells differentiated and then fused with each other to form new myofibers (Supplementary Fig. S5d, e). All of these results indicated that overactivated JAK-STAT3 signaling in adult SCs also promotes asymmetric cell fate segregation during skeletal muscle regeneration.

Our findings provide strong support that during muscle repair, the activity of JAK-STAT3 signaling in SCs is tightly regulated by the miR-31-IL34 axis to enhance 
a

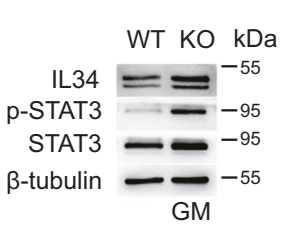

e

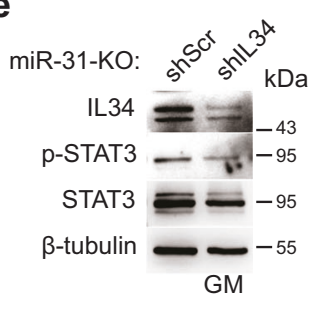

h

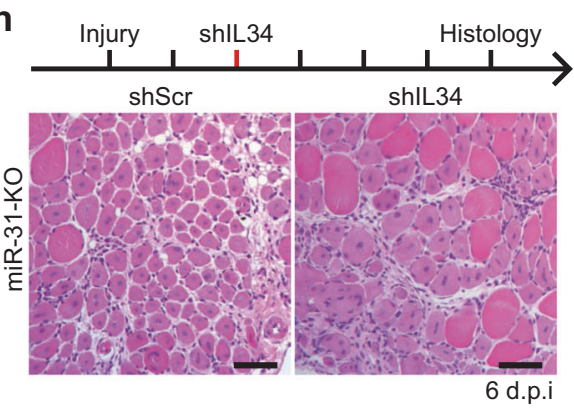

k
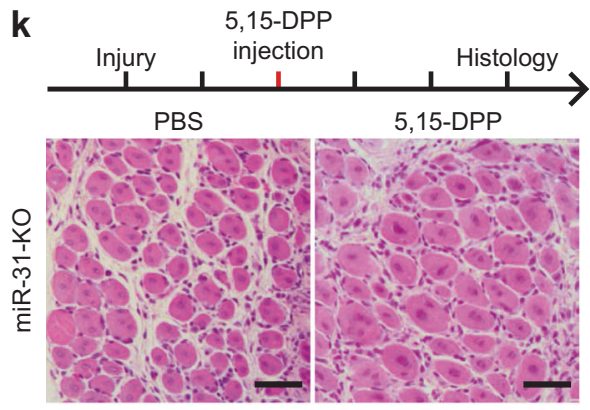

n

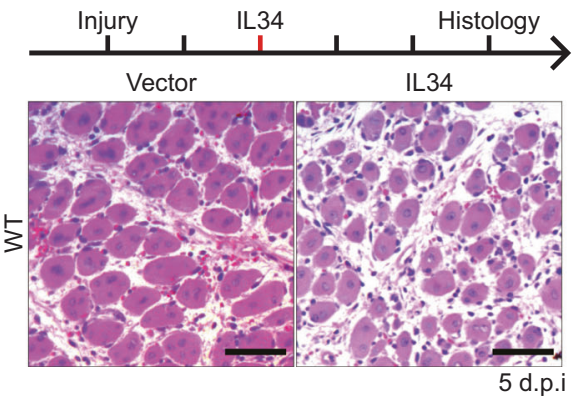

muscle repair and functional performance (Fig. 7m). Manipulation of JAK-STAT3 signaling using smallmolecule inhibitors or siRNAs/shRNA modulates the function of SCs and results in better muscle functional

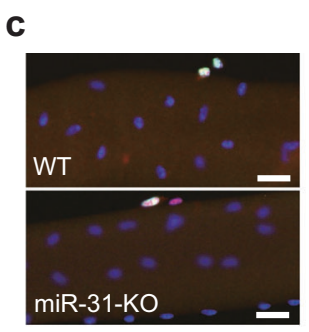

Pax7; Ki67; DAPI

f

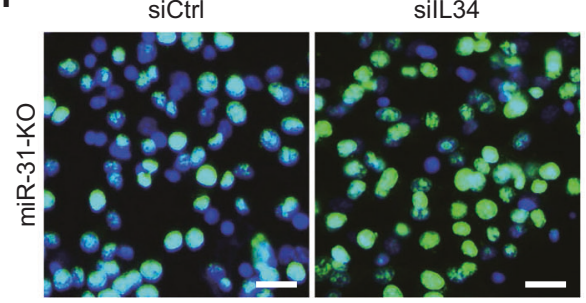

Ki67; DAPI

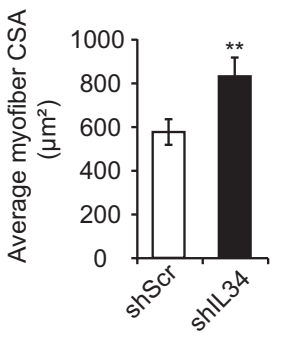

I

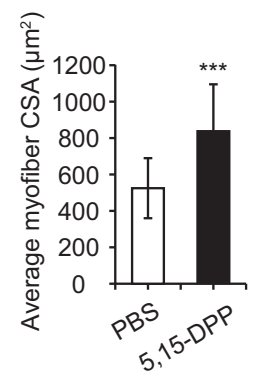

o

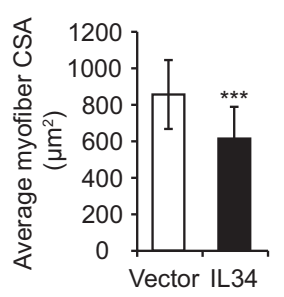

d

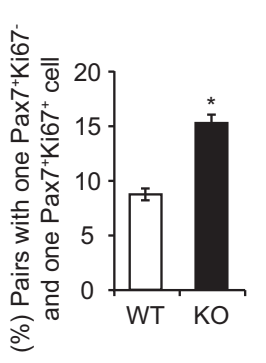

g

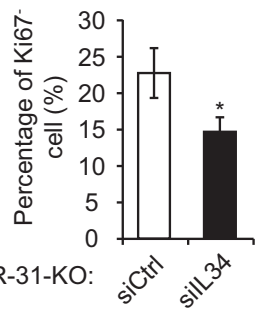

j

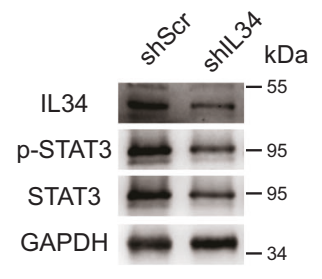

m

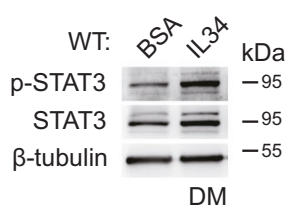

p

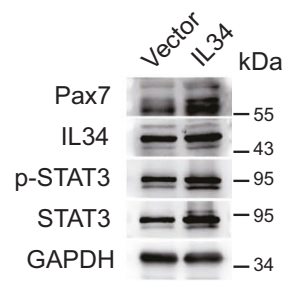

performance. Therefore, we speculate that the specifically targeted miR-31-IL34 axis is a candidate pathway that can counteract the functional exhaustion of SCs in pathological conditions. 
Fig. 6 miR-31 prevents hyperactivation of JAK-STAT3 signaling by repressing $I L 34$. a The immunoblots demonstrate the protein levels of IL34, p-STAT3, STAT3, and $\beta$-tubulin in proliferating SCs of WT and miR-31-KO mice. b Western blot analysis of MyHC, IL34, p-STAT3, STAT3, and unrelated protein (GAPDH) in 1-day differentiated cultures of WT and miR-31-KO mice. c Single myofibers were isolated from the EDL muscles of WT and miR-31-KO mice. After $42 \mathrm{~h}$ of culture, myofibers were fixed and costained for Pax7 and Ki67. Nuclei were identified by labeling with DAPI. Representative merged photomicrographs of cultured myofibers of WT and miR-31-KO mice after staining for Pax7, Ki67, and DAPI. Scale bar: $25 \mu \mathrm{m}$. d Quantification of cell doublets containing one $\mathrm{Pax} 7^{+} \mathrm{Ki} 67^{-}$cell and one $\operatorname{Pax} 7^{+} \mathrm{Ki} 67^{+}$cell among the total dividing SCs on single fibers after culturing for $42 \mathrm{~h}$. $* P<0.05$. e The representative immunoblots presented here show IL34, p-STAT3, STAT3 and $\beta$-tubulin protein levels in shIL34- and shScramble-treated proliferating SCs of miR-31-KO mice. f Representative merged images of silL34- and siControl-treated proliferating SCs of miR-31-KO mice after staining for Ki67 and DAPI. Scale bar: $20 \mu \mathrm{m}$. g Quantification of the percentage of Ki67 cells in miR-31-KO cultures after silL34 or siControl treatment. $* P<$ 0.05 . $\mathbf{h}$ Two days after injecting $1.2 \% \mathrm{BaCl}_{2}$ into the TA muscles of miR-31-KO mice, a lentivirus expressing shRNA against IL34 to downregulate IL34 mRNA of injured miR-31-KO TA muscles or shScramble was delivered to the injured muscles. Samples were collected at day 6 post injury. Representative photomicrograph of H\&Estained TA muscle sections presenting better recovery of injured TA muscles with impaired IL34 function. Scale bar: $50 \mu \mathrm{m}$. i Average size of regenerating myofibers at day 6 after injury. $* * P<0.001$. j The immunoblots presented here show the IL34, p-STAT3, STAT3, and unrelated GAPDH protein levels in regenerating miR-31-KO TA muscle after treatment with IL34 shRNA. k Two days after injecting $1.2 \% \mathrm{BaCl}_{2}$ into the TA muscles of miR-31-KO mice, a STAT3 inhibitor, 5,15 DPP, was delivered to the injured TA muscles. Histology analysis of the regenerating TA muscles of miR-31-KO mice treated with STAT3 inhibitor or vehicle control at 5 days after injury by H\&E staining. Scale bar: $50 \mu \mathrm{m}$. I Quantification of the myofiber average CSA in regenerating TA muscle 5 days after injury. $N=3$ in each group. $* * * P<0.001$. m WT proliferating SCs grown in differentiation medium were treated with or without $100 \mathrm{ng} / \mathrm{ml}$ recombinant IL34 for $24 \mathrm{~h}$. The immunoblots demonstrate the protein levels of pSTAT3, STAT3, and unrelated $\beta$-tubulin in cultures after the addition of recombinant IL34. $\mathbf{n}$ Two days after injecting $1.2 \% \mathrm{BaCl}_{2}$ into the TA muscles of WT mice, an adenovirus expressing IL34 or mCherry (control) was delivered to the injured muscles of WT mice. Samples were collected at day 5 post injury. H\&E-stained TA muscle sections show worse recovery of injured TA muscles with enhanced IL34 function. Scale bar: $50 \mu \mathrm{m}$. o Average size of regenerating myofibers at day 5 after injury. $N=3$ in each group. $* * * P<0.001$. p The immunoblots presented here illustrate the levels of Pax7, IL34, p-STAT3, STAT3, and the unrelated protein GAPDH in regenerating WT TA muscles after the overexpression of IL34

\section{Materials and methods}

\section{Animals}

The generation of the miR-31 ${ }^{-1-}$ mice on a C57BL/6 background used in this study has been previously described [51]. Housing, husbandry and all experimental protocols used in this study were performed according to the Regulations of Beijing Laboratory Animal Management and strictly obeyed the guidelines of the China Agricultural
University Laboratory Animal Welfare and Animal Experimental Ethical Inspection Form (approval number: SKLAB-2011-04-03). At 70 days of age, $75 \mu$ of $1.2 \%$ $\mathrm{BaCl}_{2}$ (Sigma-Aldrich) solution was injected into the TA muscle to cause necrotic injury for the muscle regeneration experiments. For EdU pulse-chase experiments, animals were given an intramuscular injection of EdU (100 $\mu \mathrm{g}$ per mouse) at $2 \mathrm{~h}$ before sacrifice. For in vivo inhibition of STAT3 in miR-31-KO mice, we directly injected $25 \mu$ of 5,15 DPP $(100 \mu \mathrm{M})$ into damaged TA muscles 2 days after $1.2 \% \mathrm{BaCl}_{2}$ treatment as described by Price et al. [20].

\section{Myofiber isolation and culture}

EDL muscles were carefully isolated from 10-week-old WT and miR-31-KO mice and digested with collagenase I (Sigma-Aldrich, C0130). The muscles were incubated in $0.2 \%$ collagenase I in serum-free Dulbecco's Modified Eagle Medium (DMEM) on a horizontal rocking bed at $37^{\circ} \mathrm{C}$ for $60 \mathrm{~min}$. The digested muscle was placed in a $6-\mathrm{cm}$ Petri dish precoated with horse serum, and myofibers were isolated and transferred under a microscope. For suspension culture, single myofibers were placed in a 48-well plate precoated with horse serum. Myofibers were also subjected to adherent culture by coating 48-well plates with a layer of Matrigel. For culturing, myofibers were maintained in DMEM with high glucose (Life Technologies) supplemented with $10 \%$ horse serum, $0.5 \%$ chick embryo extract, and $1 \%$ penicillin-streptomycin. The fibers were cultured at $37^{\circ} \mathrm{C}$ in a $5 \% \mathrm{CO}_{2}$ atmosphere for $42 \mathrm{~h}, 72 \mathrm{~h}$, or $120 \mathrm{~h}$. Freshly isolated fibers were fixed with $4 \%$ paraformaldehyde (PFA) for 20 min and stained for Pax7 and DAPI.

\section{SC isolation and culture}

Hindlimb muscles of 10-week-old WT and miR-31-KO mice were isolated under sterile conditions and minced to yield a muscle suspension. The tissue was then digested with collagenase II (500 units, Sigma-Aldrich, C6885) in DMEM with $5 \%$ horse serum at $37^{\circ} \mathrm{C}$ on a horizontal rocking bed for $60 \mathrm{~min}$. Digested muscle suspensions were washed and further digested with collagenase II (200 units) and dispase II (2.4 units per $\mathrm{ml}$; Roche) for $40 \mathrm{~min}$. Completely digested muscles were then filtered through a $40-\mu \mathrm{m}$ nylon cell strainer and washed to yield mononuclear-cell suspensions for cell-surface staining. Mononuclear cells were stained with CD31-PE/Cy7 (clone 390; Biolegend), CD45-FITC (clone 30-F11; BD Bioscience), Sca1-PerCp (clone D7; eBioscience), and $\alpha$-integrin-7-APC (clone $\mathrm{R} 2 \mathrm{~F} 2$; Ablabs) on ice for $60 \mathrm{~min}$. Cell storage was performed using a Beckman cell sorter equipped with lasers. Cells positive for $\alpha$-integrin-7 and negative for CD31-PE/ Cy7, CD45-FITC, and Sca1-PerCp were sorted for 
a

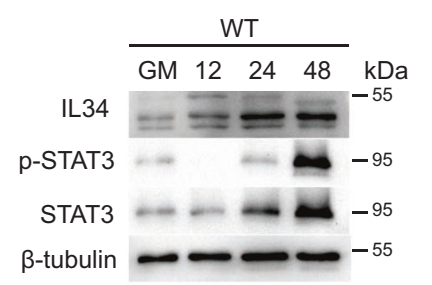

e

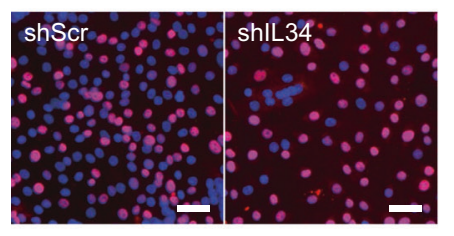

Pax7; DAPI

i

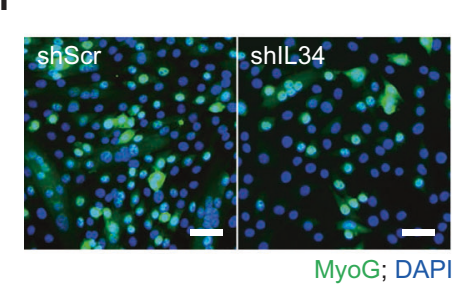

b

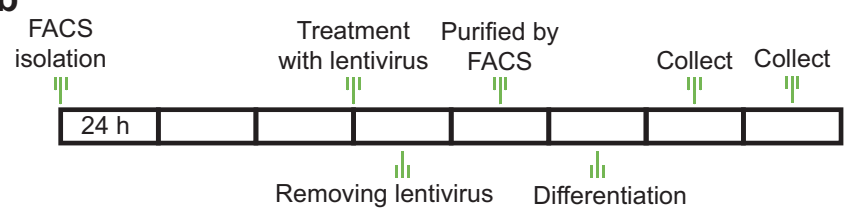

c

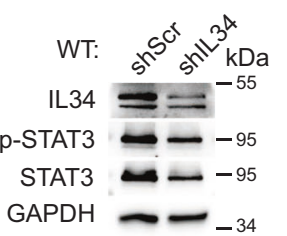

f

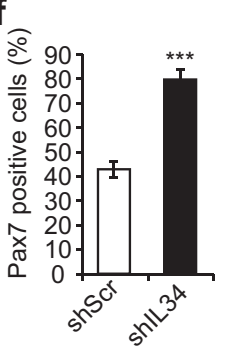

g
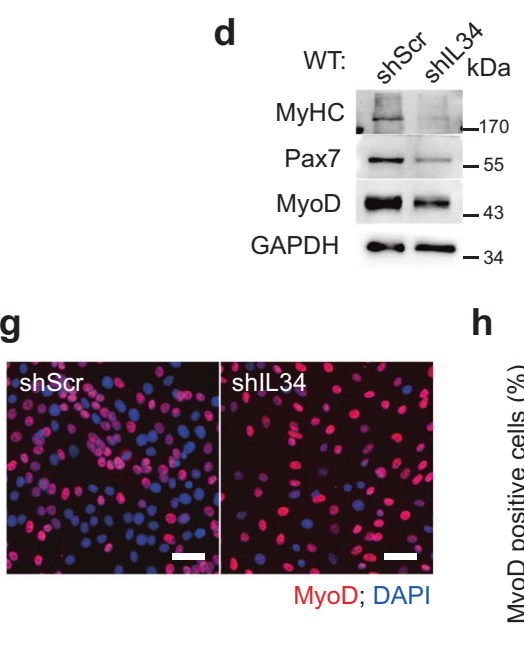

j

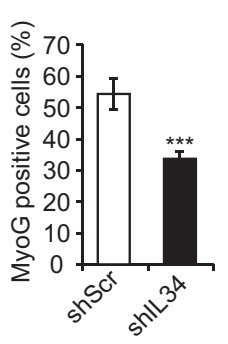

k

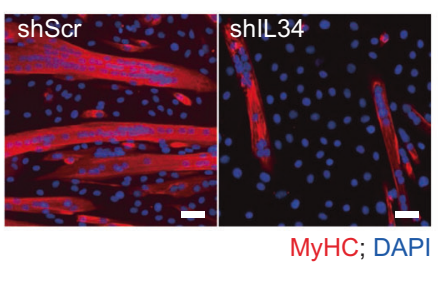

h

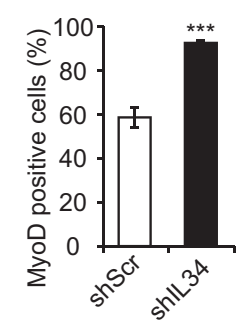

I

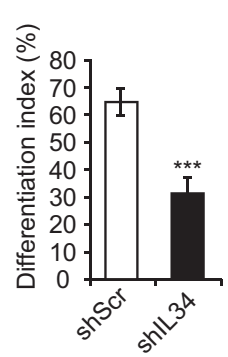

m

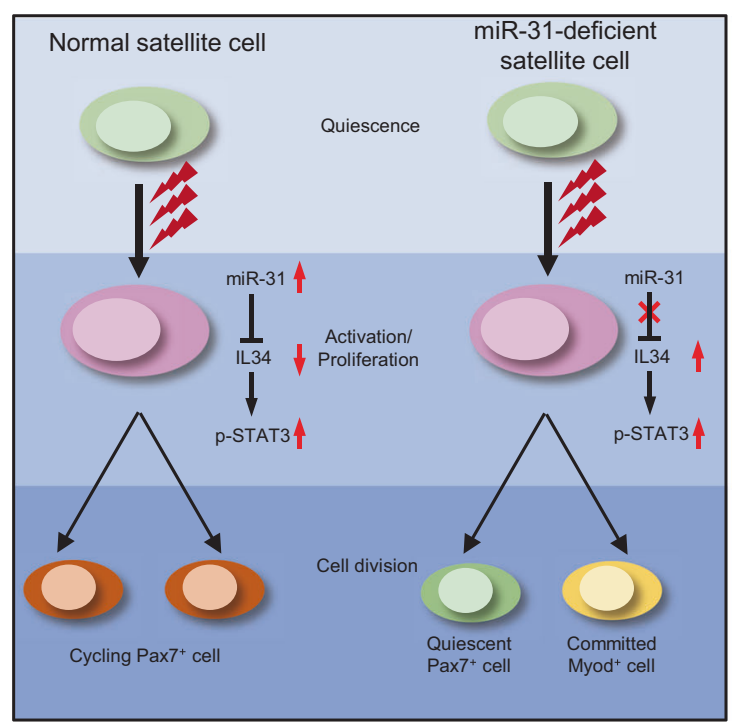

enrichment of activated SCs. Sorted SCs were cultured in growth medium (DMEM supplemented with $20 \%$ fetal bovine serum, $1 \%$ chick embryo extract, $10 \mathrm{ng} / \mathrm{ml}$ basic fibroblast growth factor and $1 \%$ penicillin-streptomycin) on $10 \%$ Matrigel-coated (BD Biosciences) cell culture plates at $37{ }^{\circ} \mathrm{C}$ in a $5 \% \mathrm{CO}_{2}$ atmosphere. To induce differentiation, 
Fig. 7 IL34 is required for myogenic lineage progression in cultured SCs. a Fresh SCs were seeded, cultured in growth medium, and then induced to differentiate for various amounts of time. The immunoblots presented here reveal the protein levels of IL34, p-STAT3, STAT3, and unrelated $\beta$-tubulin in cultured SCs at different time points after initiation of differentiation. $\mathbf{b}$ SCs were isolated from the hindlimbs of WT mice using FACS, cultured in growth medium for 3 days, and treated with shIL34 lentivirus or control shRNA (shScr) for $36 \mathrm{~h}$. The positively infected SCs were then purified by FACS according to the intrinsic enhanced green fluorescent protein (EGFP) fluorescence expressed by the lentivirus. Purified infected SCs were cultured for further analysis. c Western blot analysis of the levels of IL34, pSTAT3, STAT3, and an unrelated protein (GAPDH) in WT cell cultures after shScr and shIL34 treatment. d Western blot analysis of the levels of MyHC, MyoD, Pax7, and an unrelated protein (GAPDH) in WT cell cultures after shScr and shIL34 treatment. e Representative merged photomicrographs of SCs stably infected with a lentivirus expressing shIL34 or shScr that were induced to differentiate for one day and stained for Pax 7 and DAPI. Scale bar: $30 \mu \mathrm{m}$. f Quantification of the percentage of $\mathrm{Pax}^{+}$SCs after shScr and shIL34 infection. *** $P<0.001$. g Representative merged photomicrographs of SCs stably infected with a lentivirus expressing shIL34 or shScr that were induced to differentiate for one day and stained for MyoD, and DAPI. Scale bar: $30 \mu \mathrm{m}$. h Quantification of the percentage of $\mathrm{MyoD}^{+} \mathrm{SCs}$ after lentiviral infection. ${ }^{*} * *<0.001$. i Representative merged photomicrographs of SCs stably infected with a lentivirus expressing shIL34 or shScr that were induced to differentiate for one day and stained for MyoG and DAPI. Scale bar: $30 \mu \mathrm{m}$. j Quantification of the percentage of $\mathrm{MyoG}^{+}$SCs after lentiviral infection. $* * * P<0.001$. $\mathbf{k}$ Representative merged images of differentiated $\mathrm{MyHC}^{+}$myotubes in SCs that were stably infected with shIL34 or shScr lentivirus and analyzed $48 \mathrm{~h}$ after differentiation was induced. Scale bar: $30 \mu \mathrm{m}$. I The differentiation index was measured after shIL34 treatment according to the percentage of nuclei in MyHC-positive cells. $* * * P<$ 0.001. $\mathbf{m}$ The miR-31-IL34 axis working model in skeletal muscle regeneration

cells at $60 \%$ confluency were switched to differentiation medium containing DMED, 5\% horse serum and 1\% penicillin-streptomycin.

\section{SC transplantation}

SCs freshly isolated by FACS were initially seeded and cultured in growth medium for $72 \mathrm{~h}$, then carefully detached by treating them with $0.1 \%$ trypsin. A total of 250,000 enumerated SCs were transferred into a sterile collection tub and washed four times with cold PBS to completely remove the remaining medium. Sixty microliters of PBS was added to the resuspended cell pellet, which was immediately injected into 2-day injured TA muscles of adult WT mice.

\section{Histological and morphometric analysis}

To assess skeletal muscle morphology and regeneration, skeletal muscle groups were collected and fixed in 4\% PFA and then processed for routine paraffin histology. Fivemicron-thick transverse sections of TA muscle were subjected to $\mathrm{H} \& \mathrm{E}$ staining. For quantitative estimation, the cross-sectional area (CSA) was calculated in H\&E-stained TA muscle sections using ImageJ software.

\section{Immunofluorescence and immunohistochemistry}

For immunohistochemistry analysis, antigen retrieval was performed by heating TA muscle sections in Tris-EDTA buffer ( $\mathrm{pH}$ 9.0) in a microwave. Sections were then immunostained according to the protocol described by Tian et al. [51]. For immunofluorescence, dewaxed sections were first microwaved in Tris-EDTA buffer ( $\mathrm{pH}$ 9.0). TA muscle sections, 4\% PFA-fixed single myofibers or cultured SCs were washed with PBS and permeabilized with $0.5 \%$ Triton X-100 for $15 \mathrm{~min}$. Cells and sections were supplemented with blocking buffer (Beyotime, China) at room temperature for $1 \mathrm{~h}$ and then incubated with primary antibodies at $4{ }^{\circ} \mathrm{C}$ overnight. Sections and cells were washed briefly and incubated with secondary antibodies (Invitrogen) for $1 \mathrm{~h}$ at room temperature. DAPI staining was used to indicate nuclei. The following primary antibodies were used: mouse anti-Pax7 (1:100, Developmental Studies Hybridoma Bank (DSHB), lowa City, IA), mouse anti-MyoD1 (1:100, DAKO), rabbit anti-MyoD (1:100, Santa Cruz, sc-760), mouse anti-MyoD (1:100, Santa Cruz, sc-32758), mouse anti-embryonic Myosin Heavy Chain BF-45/F1.652 (1:40, DSHB, lowa City, IA), rabbit anti-myogenin (1:200, Santa Cruz, sc-576), rabbit anti-laminin (1:1000, Sigma-Aldrich, L9393), and rabbit anti-Ki67 (1:500, Invitrogen).

\section{miR-31 in situ hybridization}

Analysis of miR-31 in injured skeletal muscle was performed by in situ hybridization according to a previously described protocol. In brief, digoxigenin (DIG)-labeled miR-31, scrambled LNA probes (Exiqon) and the positive control U6 were incubated at $55^{\circ} \mathrm{C}$. Anti-DIG-AP antibody followed by development using BM purple substrate (Roche) were used to visualize in situ signals. The nuclei were labeled using nuclear fast red-crystal staining.

\section{Western blotting}

Total protein extracts were obtained by homogenizing skeletal muscle and cell cultures in radioimmunoprecipitation buffer (Cell Signaling Technology, Cat: 9806) supplemented with protease inhibitor cocktail (Beyotime, China) and phosphatase inhibitors (Roche). Cell debris was removed by centrifugation, and the supernatant was collected and stored at $-80^{\circ} \mathrm{C}$. Protein concentration was measured using a BCA protein assay kit (Beyotime, China), and $30 \mu \mathrm{g}$ of protein from different samples was then electrophoresed by $10 \%$ SDS-PAGE. Western blotting analysis was performed using a standard protocol. The 
following antibodies were used: Pax7 (1:500, DSHB), pSTAT3 (1:1000, CST), STAT3 (1:1000, CST), IL34 (1:1000, Abcam), MyoD (1:500, Scanta), MyoD1 (1:500, Dako), MyoG (1:1000, Scanta), GAPDH (1:10000, CST), $\beta$-tubulin (1:10000, Abcam), horseradish peroxidase (HRP)conjugated anti-mouse IgG (1:10000, CST), and HRPconjugated anti-rabbit IgG (1:10000, CST).

\section{Cell proliferation assay}

In vivo cell proliferation and SC proliferation were analyzed by labeling cells with EdU for 120 min using a Click-iT EdU Cell Proliferation Assay Kit (Invitrogen). In brief, $1.5 \times 10^{4} \mathrm{SCs}$ were seeded on Matrigel-coated glass cover slips placed in a 24 -well plate and incubated at $37^{\circ} \mathrm{C}$ in a $5 \% \mathrm{CO}_{2}$ atmosphere for five days; EdU was added to label proliferating cells during the last $2 \mathrm{~h}$. To evaluate in vivo cell proliferation, EdU was injected $2 \mathrm{~h}$ prior to harvesting regenerating TA muscles from WT and miR-31-KO mice at 3 days post injury.

\section{Microarray and data analysis}

For mRNA microarrays, total RNA samples were collected from cultured proliferating SCs of WT and miR-31-KO mice. Then, the RNA samples were submitted to Gene Denovo Biotechnology Co. Library construction, sequencing and bioinformatics analysis were performed, strictly obeying corporate standard operation protocols, which are available online (http://www.genedenovo.com/).

\section{Real-time qPCR analyses}

Total RNA from injured or uninjured TA muscle and SCs was extracted with TRIzol reagent (Invitrogen, Life Technologies) according to the manufacturer's standards. Firststrand cDNA for PCR analyses was generated with M-MLV Reverse Transcriptase (Sigma) using oligo (dT) primers. Real-time PCR analysis was performed using a Light Cycler 480 real-time PCR system (Roche). The qRT-PCR primers were as follows:

Pax7-forward: 5'-TGGGGTCTTCATCAACGGTC-3'; Pax7-reverse: 5'-ATCGGCACAGAATCTTGGAGA-3';

IL34-forward: 5'-TACAAGAACCGGCTTCAGTACA3'; IL34-reverse: 5'-GCATTGAGACTCACCAAGACC-3'; GAPDH-forward: 5'-CCCAGAAGACTGTGGATGG-3'; GAPDH-reverse: 5'-ACACATTGGGGGTAGGAACA-3'.

\section{Transfection and luciferase assays}

3'-UTR fragments of IL34 containing the miR-31 target site were cloned into the psiCHECK ${ }^{\mathrm{TM}}-2$ vector (Promega). Mutagenesis of the miR-31-binding site, cell culture, and luciferase assay were performed as previously described [52].

\section{Construction and packaging of lentivirus and adenovirus granules}

Lentivirus expressing control shRNA or shRNA against IL34 and adenovirus expressing IL34 or mCherry were designed and generated by a commercial organization. Packaging of the lentivirus and adenovirus granules was strictly performed according to the standard operating protocols of Cyagen Biosciences Inc.

\section{In vitro knockdown of IL34}

For viral transduction, quiescent SCs $\left(4 \times 10^{5}\right)$ isolated from the hind limbs of adult miR-31-KO or WT mice were plated in a 6-well culture dish for amplification culture. Then, virus was added to infect the SCs, with the quantity of the added virus regulated to ensure a multiplicity of infection of 60 . A total of $5 \mu \mathrm{g} / \mathrm{ml}$ polybrene was used to increase infection efficiency. After infection for $12 \mathrm{~h}$, the medium was removed and replaced with fresh medium, and cells were incubated for another day before purified enhanced green fluorescent protein-positive cells were isolated by FACS.

\section{In vivo knockdown and overexpression of IL34}

For overexpression of IL34 in injured TA muscle of WT mice, an adenovirus expressing IL34 or mCherry (control) in $0.9 \% \mathrm{NaCl}$ was directly injected into the injured muscle at day 2 post injury, and the muscle was then allowed to recover for another 3 days. The viral dose was $2 \times 10^{11}$ plaque-forming units (p.f.u.) per damaged TA muscle of WT mice.

For knockdown of IL34 in injured TA muscle of miR31-KO mice, a lentivirus expressing shRNA against IL34 or control in $0.9 \% \mathrm{NaCl}$ was directly delivered into the injured TA muscle of miR-31-KO mice by local injection at day 2 after $\mathrm{BaCl}_{2}$-induced injury, and muscles were collected for analysis 6 days post injury. The viral dose was $5 \times 10^{8}$ p.f.u.

\section{Statistical analysis}

A minimum of three and up to five replicates were performed for all experiments. Values are presented as the means \pm SEM. Differences between two experimental groups were tested for statistical significance using an unpaired two-tailed Student's $t$ test. $p<0.05$ was considered to be significant.

Acknowledgements This work was supported by the National Natural Science Foundation of China (31790412), the National Science 
Foundation of China (2015CB943103), the Ministry of Agriculture Transgenic Major Projects of China (2016ZX08010004), the Project for Extramural Scientists of State Key Laboratory of Agrobiotechnology (2019SKLAB6-1), and the earmarked fund for Modern Agro-Industry Technology Research Systems of China (CARS-37).

Author contributions Conceptualization, Y. S. and Q. M.; Methodology, Y. S., Y. Y., Y. Z., J. S., and C. L.; Writing-original draft, Y. S.; Investigation, T. W., K. W., T. H, L. X., C. L. and L. L.; WritingReview \& Editing, Q. M., Y. Z., Z. Y., and Y. S.; Funding Acquisition, Q. M.; Resources, Z. Y., Y. S., N. L.; Supervision, Z. Y. and Q. M.

\section{Compliance with ethical standards}

Conflict of interest The authors declare that they have no conflict of interest.

Publisher's note: Springer Nature remains neutral with regard to jurisdictional claims in published maps and institutional affiliations.

\section{References}

1. Almada AE, Wagers AJ. Molecular circuitry of stem cell fate in skeletal muscle regeneration, ageing and disease. Nat Rev Mol Cell Biol. 2016;17:267-79.

2. Mauro A. Satellite cell of skeletal muscle fibers. J Biophys Biochem Cytol 1961;9:493-5.

3. Wang YX, Rudnicki MA. Satellite cells, the engines of muscle repair. Nat Rev Mol Cell Biol. 2011;13:127-33.

4. Abou-Khalil R, Brack AS. Muscle stem cells and reversible quiescence: the role of sprouty. Cell Cycle 2010;9:2575-80.

5. Kuang S, Rudnicki MA. The emerging biology of satellite cells and their therapeutic potential. Trends Mol Med. 2008;14:82-91.

6. Yin H, Price F, Rudnicki MA. Satellite cells and the muscle stem cell niche. Physiol Rev. 2013;93:23-67.

7. Bernet JD, Doles JD, Hall JK, Kelly Tanaka K, Carter TA, Olwin BB. p38 MAPK signaling underlies a cell-autonomous loss of stem cell self-renewal in skeletal muscle of aged mice. Nat Med. 2014;20:265-71.

8. Dumont NA, Wang YX, Rudnicki MA. Intrinsic and extrinsic mechanisms regulating satellite cell function. Development 2015; 142:1572-81.

9. Hindi SM, Kumar A. TRAF6 regulates satellite stem cell selfrenewal and function during regenerative myogenesis. J Clin Invest. 2016;126:151-68.

10. Kuang S, Gillespie MA, Rudnicki MA. Niche regulation of muscle satellite cell self-renewal and differentiation. Cell Stem Cell 2008;2:22-31.

11. Rozo M, Li L, Fan CM. Targeting beta1-integrin signaling enhances regeneration in aged and dystrophic muscle in mice. Nat Med. 2016;22:889-96

12. Ogura Y, Hindi SM, Sato S, Xiong G, Akira S, Kumar A. TAK1 modulates satellite stem cell homeostasis and skeletal muscle repair. Nat Commun. 2015;6:10123.

13. Paris ND, Soroka A, Klose A, Liu W, Chakkalakal JV. Smad4 restricts differentiation to promote expansion of satellite cell derived progenitors during skeletal muscle regeneration. Elife. 2016;5:pii: e19484.

14. Chakkalakal JV, Jones KM, Basson MA, Brack AS. The aged niche disrupts muscle stem cell quiescence. Nature. 2012; 490:355-60.

15. Yue F, Bi P, Wang C, Shan T, Nie Y, Ratliff TL, et al. Pten is necessary for the quiescence and maintenance of adult muscle stem cells. Nat Commun. 2017;8:14328.
16. Kisseleva T, Bhattacharya S, Braunstein J, Schindler CW. Signaling through the JAK/STAT pathway, recent advances and future challenges. Gene 2002;285:1-24.

17. O'Shea JJ, Gadina M, Schreiber RD. Cytokine signaling in 2002: new surprises in the Jak/Stat pathway. Cell 2002;109: S121-31.

18. Schindler C, Levy DE, Decker T. JAK-STAT signaling: from interferons to cytokines. J Biol Chem. 2007;282:20059-63.

19. Wang $\mathrm{K}$, Wang $\mathrm{C}$, Xiao F, Wang H, Wu Z. JAK2/STAT2/STAT3 are required for myogenic differentiation. J Biol Chem. 2008;283:34029-36.

20. Price FD, von Maltzahn J, Bentzinger CF, Dumont NA, Yin H, Chang NC, et al. Inhibition of JAK-STAT signaling stimulates adult satellite cell function. Nat Med. 2014;20:1174-81.

21. Hirano T, Ishihara K, Hibi M. Roles of STAT3 in mediating the cell growth, differentiation and survival signals relayed through the IL-6 family of cytokine receptors. Oncogene 2000; 19:2548-56

22. Johnson DE, O'Keefe RA, Grandis JR. Targeting the IL-6/JAK/ STAT3 signalling axis in cancer. Nat Rev Clin Oncol 2018:15:234-48.

23. Morris EJ, Kawamura E, Gillespie JA, Balgi A, Kannan N, Muller WJ, et al. Stat 3 regulates centrosome clustering in cancer cells via Stathmin/PLK1. Nat Commun. 2017;8:15289.

24. Sargeant TJ, Lloyd-Lewis B, Resemann HK, Ramos-Montoya A, Skepper J, Watson CJ. Stat3 controls cell death during mammary gland involution by regulating uptake of milk fat globules and lysosomal membrane permeabilization. Nat Cell Biol. 2014; 16:1057-68.

25. Lin H, Lee E, Hestir K, Leo C, Huang M, Bosch E, et al. Discovery of a cytokine and its receptor by functional screening of the extracellular proteome. Science. 2008;320:807-11.

26. Wang Y, Szretter KJ, Vermi W, Gilfillan S, Rossini C, Cella M, et al. IL-34 is a tissue-restricted ligand of CSF1R required for the development of Langerhans cells and microglia. Nat Immunol. 2012;13:753-60.

27. Chen JF, Tao Y, Li J, Deng Z, Yan Z, Xiao X, et al. microRNA-1 and microRNA-206 regulate skeletal muscle satellite cell proliferation and differentiation by repressing Pax7. J Cell Biol. 2010;190:867-79

28. Dai Y, Wang YM, Zhang WR, Liu XF, Li X, Ding XB, et al. The role of microRNA-1 and microRNA-206 in the proliferation and differentiation of bovine skeletal muscle satellite cells. In Vitr Cell Dev Biol Anim 2016;52:27-34.

29. Liu N, Williams AH, Maxeiner JM, Bezprozvannaya S, Shelton JM, Richardson JA, et al. microRNA-206 promotes skeletal muscle regeneration and delays progression of Duchenne muscular dystrophy in mice. J Clin Invest. 2012;122:2054-65.

30. Sato T, Yamamoto T, Sehara-Fujisawa A. miR-195/497 induce postnatal quiescence of skeletal muscle stem cells. Nat Commun. 2014;5:4597.

31. Crist CG, Montarras D, Buckingham M. Muscle satellite cells are primed for myogenesis but maintain quiescence with sequestration of Myf5 mRNA targeted by microRNA-31 in mRNP granules. Cell Stem Cell 2012;11:118-26.

32. Cheung TH, Quach NL, Charville GW, Liu L, Park L, Edalati A, et al. Maintenance of muscle stem-cell quiescence by microRNA489. Nature. 2012;482:524-8.

33. Cacchiarelli D, Incitti T, Martone J, Cesana M, Cazzella V, Santini T, et al. miR-31 modulates dystrophin expression: new implications for Duchenne muscular dystrophy therapy. EMBO Rep 2011;12:136-41.

34. Ono Y, Gnocchi VF, Zammit PS, Nagatomi R. Presenilin-1 acts via Id1 to regulate the function of muscle satellite cells in a gamma-secretase-independent manner. J Cell Sci. 2009; 122:4427-38. 
35. Ono Y, Urata Y, Goto S, Nakagawa S, Humbert PO, Li TS, et al. Muscle stem cell fate is controlled by the cell-polarity protein Scrib. Cell Rep 2015;10:1135-48.

36. Murphy MM, Lawson JA, Mathew SJ, Hutcheson DA, Kardon G. Satellite cells, connective tissue fibroblasts and their interactions are crucial for muscle regeneration. Development 2011; 138:3625-37.

37. Ichiba M, Nakajima K, Yamanaka Y, Kiuchi N, Hirano T. Autoregulation of the Stat3 gene through cooperation with a cAMP-responsive element-binding protein. J Biol Chem. 1998;273:6132-8.

38. Nakajima K, Yamanaka Y, Nakae K, Kojima H, Ichiba M, Kiuchi $\mathrm{N}$, et al. A central role for Stat3 in IL-6-induced regulation of growth and differentiation in M1 leukemia cells. EMBO J. 1996;15:3651-8.

39. Reilly SN, Liu X, Carnicer R, Recalde A, Muszkiewicz A, Jayaram R, et al. Up-regulation of miR-31 in human atrial fibrillation begets the arrhythmia by depleting dystrophin and neuronal nitric oxide synthase. Sci Transl Med. 2016;8:340ra74.

40. Kim S, Lee KS, Choi S, Kim J, Lee DK, Park M, et al. NFkappaB-responsive miRNA-31-5p elicits endothelial dysfunction associated with preeclampsia via down-regulation of endothelial nitric-oxide synthase. J Biol Chem. 2018;293:18989-9000.

41. Buono R, Vantaggiato C, Pisa V, Azzoni E, Bassi MT, Brunelli S, et al. Nitric oxide sustains long-term skeletal muscle regeneration by regulating fate of satellite cells via signaling pathways requiring Vangl2 and cyclic GMP. Stem Cells 2012;30:197-209.

42. Baek JH, Zeng R, Weinmann-Menke J, Valerius MT, Wada Y, Ajay AK, et al. IL-34 mediates acute kidney injury and worsens subsequent chronic kidney disease. J Clin Invest. 2015;125:3198-214.

43. Zwicker S, Martinez GL, Bosma M, Gerling M, Clark R, Majster $\mathrm{M}$, et al. Interleukin 34: a new modulator of human and experimental inflammatory bowel disease. Clin Sci (Lond) 2015;129:281-90.

44. Baghdadi M, Wada H, Nakanishi S, Abe H, Han N, Putra WE, et al. Chemotherapy-induced IL34 enhances immunosuppression by tumor-associated macrophages and mediates survival of shemoresistant lung cancer cells. Cancer Res 2016;76:6030-42.

45. Franze E, Dinallo V, Rizzo A, Di Giovangiulio M, Bevivino G, Stolfi C, et al. Interleukin-34 sustains pro-tumorigenic signals in colon cancer tissue. Oncotarget 2018;9:3432-45.

46. Troy A, Cadwallader AB, Fedorov Y, Tyner K, Tanaka KK, Olwin BB. Coordination of satellite cell activation and selfrenewal by Par-complex-dependent asymmetric activation of p38alpha/beta MAPK. Cell Stem Cell 2012;11:541-53.

47. McCaffrey LM, Montalbano J, Mihai C, Macara IG. Loss of the Par3 polarity protein promotes breast tumorigenesis and metastasis. Cancer Cell 2012;22:601-14.

48. Tierney MT, Aydogdu T, Sala D, Malecova B, Gatto S, Puri PL, et al. STAT3 signaling controls satellite cell expansion and skeletal muscle repair. Nat Med. 2014;20:1182-6.

49. Doles JD, Olwin BB. The impact of JAK-STAT signaling on muscle regeneration. Nat Med. 2014;20:1094-5.

50. Sampath SC, Sampath SC, Ho ATV, Corbel SY, Millstone JD, Lamb J, et al. Induction of muscle stem cell quiescence by the secreted niche factor Oncostatin M. Nat Commun. 2018; 9:1531.

51. Tian Y, Ma X, Lv C, Sheng X, Li X, Zhao R, et al. Stress responsive miR-31 is a major modulator of mouse intestinal stem cells during regeneration and tumorigenesis. Elife. 2017;6:pii: e29538.

52. Liu N, Bezprozvannaya S, Williams AH, Qi X, Richardson JA, Bassel-Duby R, et al. microRNA-133a regulates cardiomyocyte proliferation and suppresses smooth muscle gene expression in the heart. Genes Dev 2008;22:3242-54. 